\title{
Chromospheric Heating due to Cancellation of Quiet Sun Internetwork Fields
}

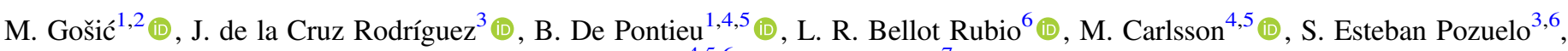 \\ A. Ortiz ${ }^{4,5,6}$ (D), and V. Polito ${ }^{7}$ (i) \\ ${ }^{1}$ Lockheed Martin Solar and Astrophysics Laboratory, Palo Alto, CA 94304, USA; mgosic@1msal.com \\ ${ }^{2}$ Bay Area Environmental Research Institute, Moffett Field, CA 94035, USA \\ ${ }^{3}$ Institute for Solar Physics, Department of Astronomy, Stockholm University, AlbaNova University Centre, SE-106 91, Stockholm, Sweden \\ ${ }^{4}$ Institute of Theoretical Astrophysics, University of Oslo, P.O. Box 1029 Blindern, NO-0315 Oslo, Norway \\ ${ }^{5}$ Rosseland Centre for Solar Physics, University of Oslo, P.O. Box 1029 Blindern, NO-0315 Oslo, Norway \\ ${ }^{6}$ Instituto de Astrofísica de Andalucía (IAA-CSIC), Apdo. 3004, E-18080 Granada, Spain \\ ${ }^{7}$ Smithsonian Astrophysical Observatory, 60 Garden Street, MS 58, Cambridge, MA 02138, USA \\ Received 2017 December 15; revised 2018 February 12; accepted 2018 February 20; published 2018 April 11
}

\begin{abstract}
The heating of the solar chromosphere remains one of the most important questions in solar physics. Our current understanding is that small-scale internetwork (IN) magnetic fields play an important role as a heating agent. Indeed, cancellations of IN magnetic elements in the photosphere can produce transient brightenings in the chromosphere and transition region. These bright structures might be the signature of energy release and plasma heating, probably driven by the magnetic reconnection of IN field lines. Although single events are not expected to release large amounts of energy, their global contribution to the chromosphere may be significant due to their ubiquitous presence in quiet Sun regions. In this paper, we study cancellations of IN elements and analyze their impact on the energetics and dynamics of the quiet Sun atmosphere. We use high-resolution, multiwavelength, coordinated observations obtained with the Interface Region Imaging Spectrograph and the Swedish $1 \mathrm{~m}$ Solar Telescope (SST) to identify cancellations of IN magnetic flux patches and follow their evolution. We find that, on average, these events live for $\sim 3$ minutes in the photosphere and $\sim 12$ minutes in the chromosphere and/or transition region. Employing multi-line inversions of the $\mathrm{Mg}$ II $h$ and $k$ lines, we show that cancellations produce clear signatures of heating in the upper atmospheric layers. However, at the resolution and sensitivity accessible to the SST, their number density still seems to be one order of magnitude too low to explain the global chromospheric heating.
\end{abstract}

Key words: Sun: atmosphere - Sun: chromosphere - Sun: magnetic fields - Sun: transition region

Supporting material: animations

\section{Introduction}

Outside of sunspots and active regions, the so-called quiet Sun (QS) is pervaded by strong kilo-Gauss fields located at the boundaries of supergranular cells-the photospheric network (NE). In between the NE, there are small and highly transient internetwork (IN) fields.

Recent results by Gošić et al. (2016) proposed IN fields as essential contributors to solar magnetism (see also Trujillo Bueno et al. 2004). The authors showed that IN elements bring magnetic flux to the solar surface at an enormous rate of $120 \mathrm{Mx} \mathrm{cm}^{-2}$ day $^{-1}$, much higher than active regions (1 Mx cm ${ }^{-2}$ day $^{-1}$; Thornton \& Parnell 2010). Part of that flux is dragged by convective motions toward the closest intergranular lanes (see, e.g., Martínez González \& Bellot Rubio 2009) and then toward the NE (Livingston \& Harvey 1975; Zirin 1985; Wang et al. 1987; Orozco Suárez et al. 2012b), providing as much unsigned magnetic flux as is present in the NE in only about $10 \mathrm{hr}$ (Gošić et al. 2014). The rest of the flux disappears through in situ fading or cancels with opposite-polarity IN patches before reaching the NE. According to Gošić et al. (2016), fading and IN flux transfer to the NE are the dominant flux removal processes in supergranular cells. However, being ubiquitous, small-scale cancellations driven by granular and supergranular flows may hold the key to deciphering a fundamental problem in solar physics, namely, chromospheric and coronal heating (e.g., Longcope \& Kankelborg 1999; Priest et al. 2002; Galsgaard \& Parnell 2005).
Therefore, it is important to understand how the cancellation of IN elements affects the upper solar atmosphere.

The cancellation of magnetic flux can take place at the junction of intergranular (Kubo et al. 2010) and mesogranular lanes (see Figure 7 in Requerey et al. 2017), and at the borders of supergranular cells where persistent sinks are observed (Requerey et al. 2018). It occurs when two opposite-polarity features come into close proximity (Livi et al. 1985; Martin 1988), leading to flux removal from the solar surface by either the submergence of an $\Omega$-shaped loop below the photosphere or the ascent of a U-shaped loop into the chromosphere (see Figure 2 in Zwaan 1987). In both cases, the observational signatures in longitudinal magnetograms are the same: two opposite-polarity patches approach each other, decreasing in size and strength, and eventually disappear below the detection limit. When the two canceling patches are part of the same magnetic system, the cancellation represents the submergence of a magnetic loop below the surface. This process is known as flux retraction. If the canceling patches were not previously connected by field lines (they are not part of the same system), the cancellation is the result of magnetic reconnection (Priest 1987; Zwaan 1987). When reconnection occurs below the surface, the opposite-polarity patches get connected by the emerging U-shaped loops (Spruit et al. 1987) and no thermal effects can be observed. If it happens above the surface, the patches get connected by a submerging $\Omega$ loop, and the energy released may produce a local brightening in the upper atmosphere. 
Using longitudinal magnetograms from Big Bear Solar Observatory, Zhang et al. (1998) estimated from the lifetimes and cancellation rate of IN elements that the total energy released through IN cancellations is $\sim 2 \times 10^{5} \mathrm{erg} \mathrm{cm}^{-2} \mathrm{~s}^{-1}$, which is comparable to the energy required to heat the corona $(3 \times$ $10^{5} \mathrm{erg} \mathrm{cm}^{-2} \mathrm{~s}^{-1}$; Withbroe \& Noyes 1977). This result is supported by Zhou et al. (2010), who used the same method in a high-resolution magnetogram sequence recorded with the Narrowband Filter Imager (NFI; Tsuneta et al. 2008) aboard the Hinode satellite (Kosugi et al. 2007). Recently, Meyer et al. (2013) used nonlinear force-free field extrapolations driven by a time series of magnetograms taken with the Helioseismic and Magnetic Imager (HMI; Scharmer et al. 2008) on board the Solar Dynamics Observatory (SDO; Pesnell et al. 2012) to study the build-up, storage, and dissipation of the magnetic energy in the QS $(\mathrm{IN}+\mathrm{NE})$ corona. They concluded that the rate of energy dissipation (lower limit of $8.7 \times 10^{4} \mathrm{erg} \mathrm{cm}^{-2} \mathrm{~s}^{-1}$ ) is roughly in agreement with the radiative losses of the QS corona. However, it is unclear whether any significant atmospheric heating (let alone coronal heating) is associated with IN cancellations, which is one of the reasons we undertake the current study.

On the other hand, Wiegelmann et al. (2013) investigated the temporal evolution of the magnetic connectivity in the QS and concluded that the energy released by reconnection processes is not sufficient to heat the chromosphere and corona. To that purpose, the authors used high temporal and spatial resolution observations from the Imaging Magnetograph eXperiment (IMaX; Martínez Pillet et al. 2011) on board the SUNRISE balloon-borne observatory (Solanki et al. 2010; Barthol et al. 2011) to extrapolate magnetic fields above the photosphere under the potential field approximation. Chitta et al. (2014) came to the same conclusion. These authors carried out magnetofrictional extrapolations of QS magnetic fields and argued that reconnections between QS magnetic elements cannot sustain the chromospheric and coronal heating.

The discrepancy between these results is likely due to the different instruments, techniques, and assumptions under which magnetic field extrapolations are carried out. For example, from magnetogram measurements only, it is not possible to know whether IN cancellations seen in the photosphere have an impact on the upper solar atmosphere. Regarding magnetic extrapolation techniques, potential (current-free) field studies provide a general idea of the global connectivity between magnetic elements, but ignore the possibility that QS magnetic fields may have a significant non-potential component (Woodard \& Chae 1999; Zhao et al. 2009). Nonlinear forcefree field extrapolations, such as the magnetofrictional method, are affected by boundary conditions. Depending on how magnetograms are preprocessed, the contribution from smallscale IN magnetic fields may be completely lost. Furthermore, there are indications that QS coronal magnetic fields are not force-free (Schrijver \& van Ballegooijen 2005), therefore results obtained by magnetofrictional modeling of the QS must be analyzed very carefully, as noted by Chitta et al. (2014).

To determine whether IN magnetic fields can provide sufficient energy to maintain chromospheric and coronal heating, it is essential to perform simultaneous, multi-instrument, multiwavelength observations of IN regions with the highest sensitivity and resolution possible. To achieve this goal, in this paper we use space observations obtained with the Interface Region Imaging Spectrograph (IRIS; De Pontieu et al. 2014), combined with ground-based observations acquired with the
Swedish $1 \mathrm{~m}$ Solar Telescope (SST; Scharmer et al. 2003). These instruments give us the opportunity to study the evolution of the weak and small IN fields at high spatial, spectral, and temporal resolution, from the photosphere up to the transition region. We track canceling IN magnetic elements in the photosphere and examine their effects on the upper atmospheric layers. From imaging and spectroscopic measurements, we will detect signatures of chromospheric heating and estimate the total energy released through cancellations of IN elements.

The observations used in this paper are presented in Section 2. We explain how cancellations are identified and tracked in Section 3. Section 4 provides examples of cancellation events, a statistical analysis, and an estimation of the total energy released through these processes. Finally, Section 5 summarizes our findings and conclusions.

\section{Observations and Data Processing}

The observations employed in this work were obtained on 2014 May 16 starting at 07:23:41 UT. They consist of coordinated IRIS data sequences and SST measurements showing the evolution of QS fields at the disk center under good seeing conditions. More precisely, the target was a supergranular cell at the disk center containing small IN patches and a strong negative-polarity NE structure at the cell boundary.

\subsection{IRIS Observations}

IRIS provides spectra in three passbands, in the nearultraviolet band (NUV) from 2783 to $2834 \AA$ and in the farultraviolet, both from 1332 to $1358 \AA$ (FUV 1), and from 1389 to $1407 \AA$ (FUV 2). This makes it possible to probe different layers of the solar atmosphere: the photosphere, the chromosphere, the upper chromosphere/lower transition region, and the transition region, respectively. In addition, IRIS is capable of recording slit-jaw images (SJI) using filters centered on Mg II $k 2796 \AA$ (SJI 2796), the far Mg II h wing at $2832 \AA$ (SJI 2832), C II $1330 \AA$ (SJI 1330), and Si IV $1400 \AA$ (SJI 1400). The former two SJIs contain contributions from the upper photosphere (2832 $\AA$ ) and from the upper photosphere to the upper chromosphere $(2796 \AA)$. For more details, we refer the reader to the first two papers in this series on the formation of IRIS diagnostics (Leenaarts et al. 2013a, 2013b). The SJI 1400 filter is sensitive to emission from the transition region Si IV $1394 / 1403 \AA$ lines and the continuum formed in the upper photosphere/lower chromosphere. Distinguishing between these two contributions can be challenging and requires the analysis of multi-instrument, multiwavelength observations (Martínez-Sykora et al. 2015). However, whenever a given region of the solar disk is not covered by the IRIS slit (i.e., there are no available spectra), some other methods have to be used. One possible approach to separate the two contributions, based on the temporal evolution of the SJI 1400 bright features, is described in Section 3.3. The SJI 1330 filter is dominated by the C II 1334/1335 $\AA$ lines formed in the upper chromosphere/ lower transition region and the continuum formed in the upper photosphere/lower chromosphere (Rathore \& Carlsson 2015).

Our observations are very sensitive, medium sparse two-step raster data taken from 07:58:54 until 11:05:32 UT without solar rotation tracking. The cadence of the spectral observations was $18.6 \mathrm{~s}$ (9.3 s per raster step) while the exposure time of individual raster scans was $8 \mathrm{~s}$. The slit sampled a quiet region of 0 ." $33 \times 60^{\prime \prime}$ with $1^{\prime \prime}$ as the raster step size. At the same time, 

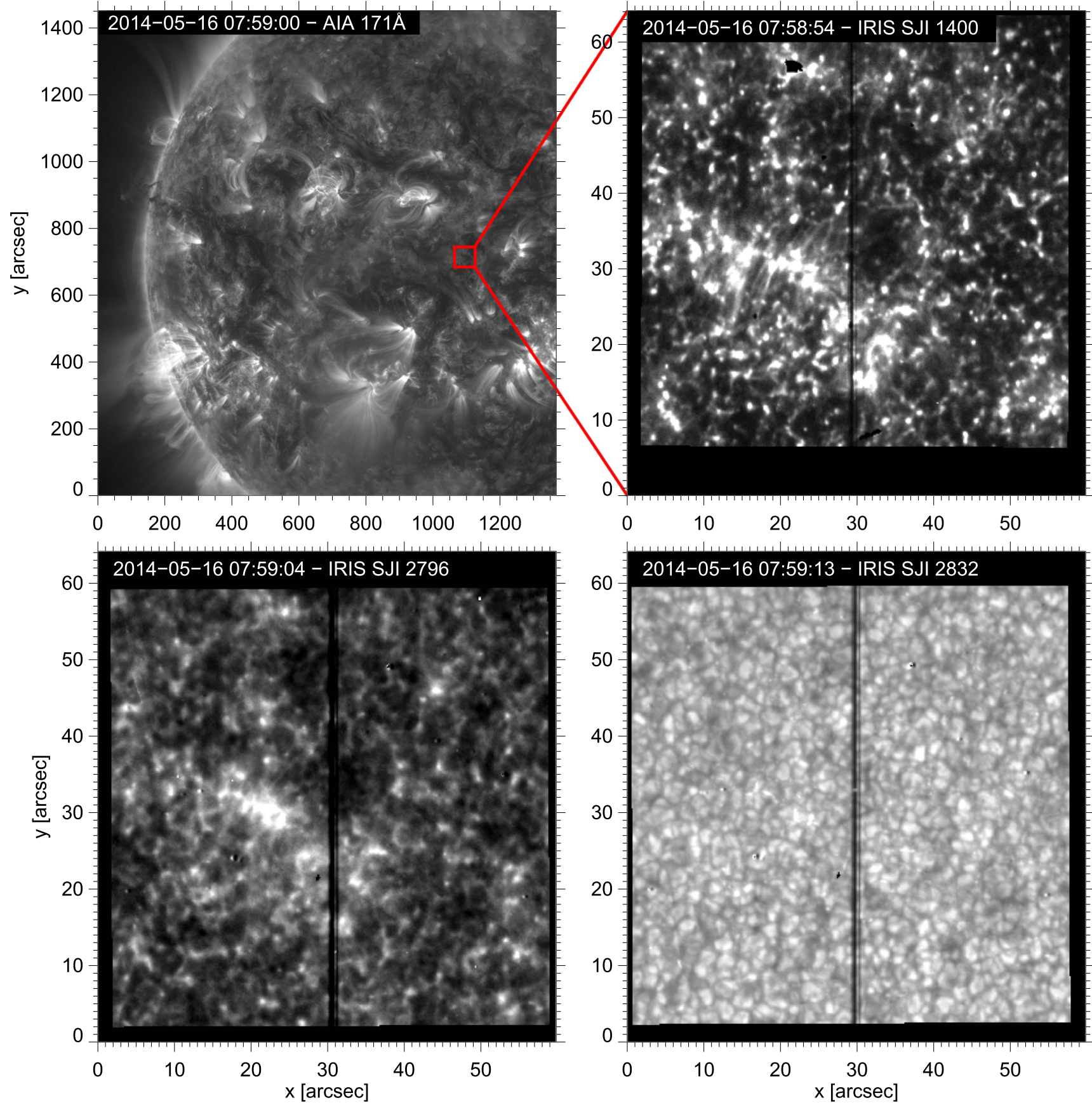

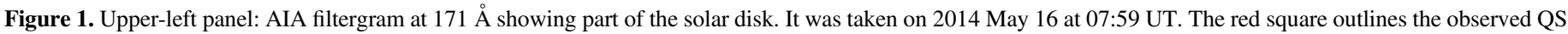
region that is blown up in the upper-right and the lower panels, displaying QS features visible in the slit-jaw images SJI 1400 , SJI 2796 and SJI 2832.

we took slit-jaw images (spatial pixel size is 0 ". 16) using the $1400 \AA$ and $2796 \AA$ filters every $\sim 19$ s. Every sixth $1400 \AA$ frame was replaced by a slit-jaw image at $2832 \AA$, giving a cadence for that wavelength of $112 \mathrm{~s}$. Those properties make the data ideal for studying the highly dynamical IN magnetic fields. The observed QS area with its surroundings can be seen in Figure 1. The upper-left panel provides the context and shows the Sun as seen at $171 \AA$ with the Atmospheric Imaging Assembly (AIA; Lemen et al. 2012) on board SDO. The other three panels show the slit-jaw images SJI 1400, SJI 2796, and SJI 2832 of the observed QS region.

The data used here are IRIS level 2 data, meaning that dark current and flat-field corrections, and geometric and wavelength calibrations have been applied. We also performed absolute calibration of the IRIS spectra. It converts the measured intensities given in units of data number per second $(\mathrm{DN} / \mathrm{s})$ into absolute intensities expressed in $\operatorname{erg~s}^{-1} \mathrm{~cm}^{-2} \mathrm{sr}^{-1} \AA^{-1}$, which can be transformed into $\mathrm{nW} \mathrm{m} \mathrm{m}^{-2} \mathrm{sr}^{-1} \mathrm{~Hz}^{-1}$. For a detailed description of the calibration process, we refer the reader to the IRIS user guide. ${ }^{8}$

\subsection{SST Observations}

The IRIS data are accompanied by coordinated observations taken at the SST with the CRisp Imaging SpectroPolarimeter

8 http://iris.lmsal.com/itn26/ 
Table 1

Description of the SST Observations

\begin{tabular}{lcccc}
\hline \hline & Fe I & $\mathrm{Mg} \mathrm{I} \mathrm{b}_{2}$ & $\mathrm{Ca} \mathrm{II}$ & \\
& $6173 \AA$ & $5173 \AA$ & $8542 \AA$ & $\mathrm{H} \alpha 6563 \AA$ \\
\hline$g_{\text {eff }}$ & 2.5 & 1.75 & 1.1 & 1.05 \\
$N_{\lambda}$ & 12 & 10 & 18 & 21 \\
$\Delta \lambda(\mathrm{m} \AA)$ & 28 & 100,50 & 100 & 100 \\
Continuum & +532 & -700 & +2400 & $\cdots$ \\
$\quad$ point $(\mathrm{m} \AA)$ & & & & \\
$n$ & 14 & 9 & 9 & 8 \\
$t_{\text {scan }}(\mathrm{s})$ & 20 & 11 & 19 & 5 \\
\hline
\end{tabular}

Note. For each observed spectral region, we list their effective Landé $g$ factors $\left(g_{\text {eff }}\right)$, number of wavelengths $\left(N_{\lambda}\right)$ and their positions with respect to the corresponding resting line centers $(\Delta \lambda)$, continuum point, number of accumulations $(n)$, and scan times $\left(t_{\text {scan }}\right)$.

(CRISP; Löfdahl 2002; Scherrer et al. 2012). CRISP is a FabryPérot filtergraph designed to obtain monochromatic images of the solar surface in selected spectral lines, from 5000 to $8600 \AA$. It measured the polarization state of the light with a polarimeter consisting of two nematic liquid crystal variable retarders and a polarizing beam splitter located in front of the cameras.

A special observing sequence was prepared to ensure complete coverage of the lower solar atmosphere while reaching the highest polarimetric sensitivity possible. To that end, the capabilities of the SST were pushed to the limit. We performed full Stokes measurements in Fe I $6173 \AA$, Mg I $b_{2}$ $5173 \AA$, and Ca II $8542 \AA$, together with non-polarimetric scans through $\mathrm{H} \alpha 6563 \AA$. These lines were chosen to sample the lower and upper photosphere, and the lower and upper chromosphere, respectively.

The effective exposure times were increased with respect to the standard values to attain higher signal-to-noise ratios, which is especially important in the QS where magnetic fields are weak-both in the photosphere and in the chromosphere. We performed a Fe I 6173 scan of 20 s duration (11 wavelength positions in steps of $28 \mathrm{m \AA}$, plus a continuum point at $+532 \mathrm{~mA}$ ), followed by a $\mathrm{Mg}$ I $b_{2}$ scan lasting $11 \mathrm{~s}$ (seven wavelength positions in steps of $100 \mathrm{~m} \AA$, plus two additional points at $\pm 50 \mathrm{~m} \AA$ from the line core and a continuum point at $-700 \mathrm{~mA}$ ), a Ca II 8542 scan of 19 s duration (17 wavelength positions at steps of $100 \mathrm{~m} \AA$ plus a continuum point at $+2.4 \AA$ ), and a short $\mathrm{H} \alpha$ scan of only $5 \mathrm{~s}$ (21 spectral positions at steps of $100 \mathrm{m \AA}$, taking only intensity filtergrams). The observations consist of 211 such cycles with a cadence of $55 \mathrm{~s}$, for a total duration of $3 \mathrm{hr}$. These data allow us to track IN magnetic elements both in the photosphere and chromosphere with unprecedented accuracy. Details of the observing sequence are summarized in Table 1.

Examples of the SST observations are shown in Figure 2. The pixel size is $0 . \prime 057$, sufficient to critically sample the diffraction limit of 0 ". 16 at $6300 \AA$. The seeing conditions were excellent for the most part, with $r_{0}$ values of up to $50 \mathrm{~cm}$, and the SST adaptive optics system helped acquire a very stable time sequence. The observations were taken from 07:23:41 to 10:28:44 UT and monitored the same QS area. Therefore, the temporal overlap between the SST and IRIS is $2.5 \mathrm{hr}$ with a continuously decreasing common field of view from roughly $50^{\prime \prime} \times 50^{\prime \prime}$ to $28^{\prime \prime} \times 50^{\prime \prime}$, as shown in Figure 3 .

The observations were reduced using the CRISPRED pipeline (de la Cruz Rodríguez et al. 2015b) and the images restored by means of the Multi-Object, Multi-Frame BlindDeconvolution techique (MOMFBD; van Noort et al. 2005). Residual seeing motions were corrected by employing the cross-correlation method of Henriques (2012). The pipeline includes additional corrections by van Noort \& Rouppe van der Voort (2008) and Shine et al. (1994). To remove instrumental polarization, a telescope polarization model was used. Finally, residual crosstalk from $I$ to $Q, U$, and $V$ was corrected using the Stokes parameters recorded in the continuum.

Longitudinal magnetograms $\mathrm{M}$ were computed from the observed Stokes profiles as

$$
\mathrm{M}=\frac{1}{2}\left(\frac{V_{\text {blue }}}{I_{\text {blue }}}-\frac{V_{\text {red }}}{I_{\text {red }}}\right),
$$

where "blue" refers to the measurements in the blue wing of the line and "red" to those in the red wing. Thus, to first order, the magnetograms are independent of line-of-sight (LOS) velocities. For example, to construct Mg 5173 magnetograms, we used filtergrams at $\pm 200 \AA$ from the line core. The magnetogram signal $\mathrm{M}$ was transformed into longitudinal magnetic flux density $\phi$ using the weak-field approximation,

$$
\phi=-\frac{1}{C} \frac{I(\lambda)}{d I(\lambda) / d \lambda},
$$

where $C=4.67 \times 10^{-3} g_{\text {eff }} \lambda_{0}^{2}, \lambda_{0}$ is the central wavelength (in $\AA$ ), and $g_{\text {eff }}$ is the effective Landé factor. The numerical values of $I(\lambda)$ and $d I(\lambda) / d \lambda$ were derived from the average QS intensity profile. To complement the longitudinal magnetograms, we also computed maps of the linear polarization signal as $\mathrm{LP}=\sum_{i=1}^{4} \sqrt{Q\left(\lambda_{i}\right)^{2}+U\left(\lambda_{i}\right)^{2}} / I\left(\lambda_{i}\right) / 4$, taking into account the first four wavelengths.

For all lines, Dopplergrams were constructed as the difference between the blue and red wing intensities divided by their sum, i.e.,

$$
D=\frac{I_{\text {blue }}-I_{\text {red }}}{I_{\text {blue }}+I_{\text {red }}}
$$

This method provides a qualitative estimate of the LOS velocity, even for the Ca II 8542 line, which may show complex shapes when it turns into emission.

The alignment of the various data sets was carried out as follows. First, the IRIS slit-jaw images were compensated for solar rotation and scaled up to match the SST pixel size. We then aligned the IRIS and SST observations using prominent NE features and bright points in the SST Fe I 6173 continuum intensity and IRIS SJI 2832 images. Since both channels practically show the same photospheric structures, the accuracy of the alignment is on the order of the IRIS pixel size. The other SST and IRIS channels were aligned to these two channels. Finally, the SST data and the IRIS SJI 1400 and SJI 2832 images were interpolated in time by applying the nearest neighbor method of interpolation to match the cadence of the SJI 2796 images (19s).

We made extensive use of CRISPEX (Vissers \& Rouppe van der Voort 2012) to visualize, analyze, and interpret the data. CRISPEX is part of the IDL SolarSoft package. ${ }^{9}$

\footnotetext{
9 SolarSoft is a set of integrated software libraries, databases, and system utilities that provide a common programming and data analysis environment for solar physics and can be accessed at http://www.lmsal.com/solarsoft/.
} 

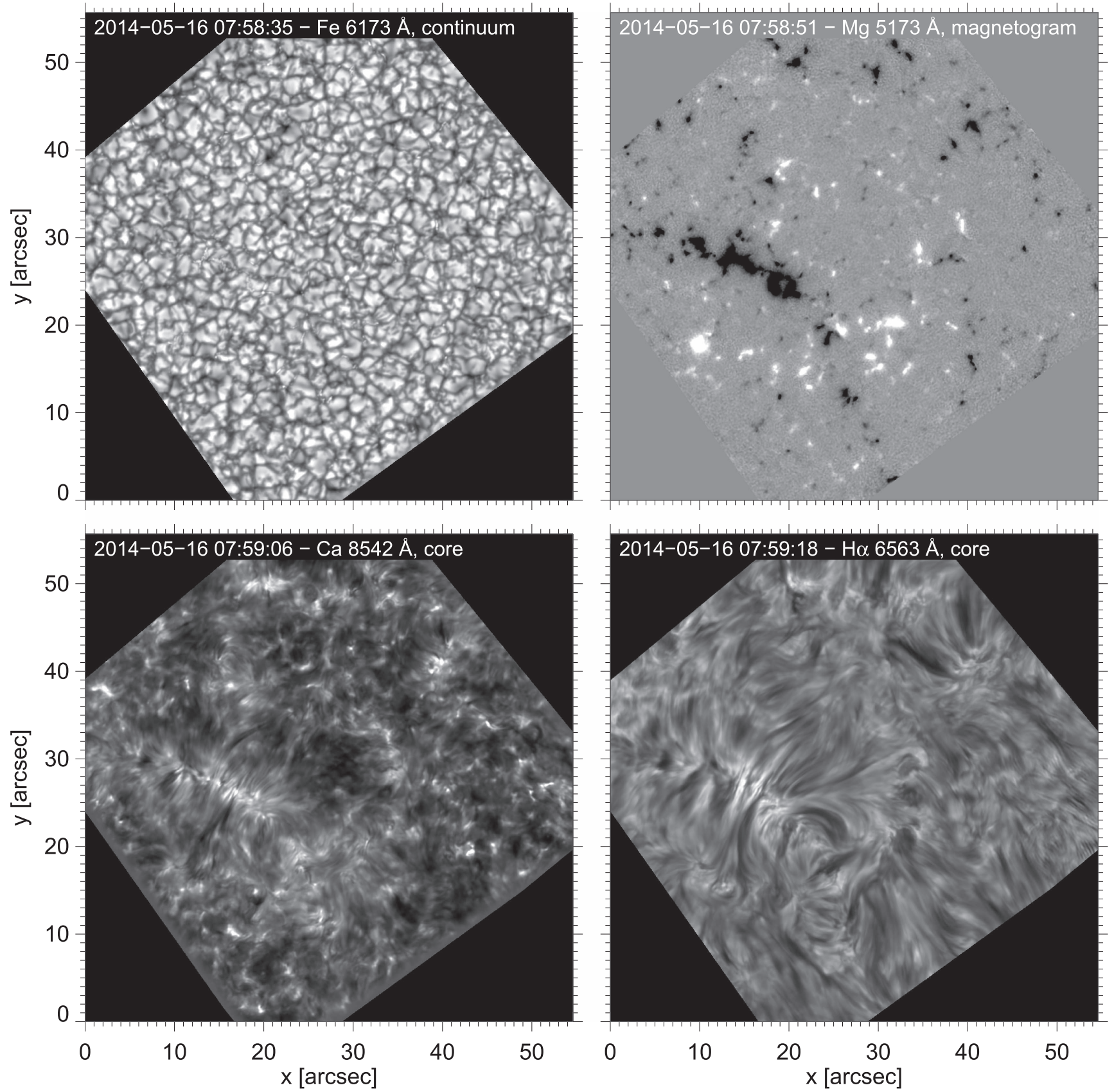

Figure 2. Examples of the continuum intensity map at $6173 \AA$ (upper left), magnetogram calculated from $\mathrm{Mg} 5173 \AA$ Stokes $I$ and $V$ filtergrams at $\pm 200 \mathrm{~m} \AA$ (upper right, scaled between $\pm 150 \mathrm{Mx} \mathrm{cm}^{-2}$ ), and intensity maps in the core of the Ca II $8542 \AA$ line (lower left) and H $\alpha 6563 \AA$ line (lower right). The observations were taken on 2014 May 16 at approximately 07:59 UT.

\section{Method}

To understand how cancellations of small-scale IN fields are coupled to the dynamics and energetics of the upper solar atmosphere, we have detected and tracked all of the individual magnetic elements visible in the photospheric data. From these patches, we identified and selected only those involved in cancellation processes and analyzed how they affect the chromosphere and corona. To that aim, we also retrieved various atmospheric parameters by employing an inversion code, that we later used to estimate the energy budget of the cancellation events. We described below each of these steps in detail.

\subsection{Identification and Tracking of Magnetic Features in SST Magnetograms}

To determine the history of individual flux features in the solar photosphere, the most suitable SST magnetograms are those constructed from Stokes $I$ and $V$ filtergrams taken at $\pm 200 \mathrm{~m} \AA$ from the core of the $\mathrm{Mg} 5173 \AA$ line. Individual elements were automatically identified and tracked using the YAFTA code (Welsch \& Longcope 2003), applying the clumping method. The adopted magnetogram signal threshold is $3 \sigma$, where $\sigma$ $\left(8 \mathrm{Mx} \mathrm{cm}^{-2}\right)$ is the standard deviation calculated in a region without clear solar signals. We consider as real magnetic concentrations only those with a minimum size of 16 pixels. 


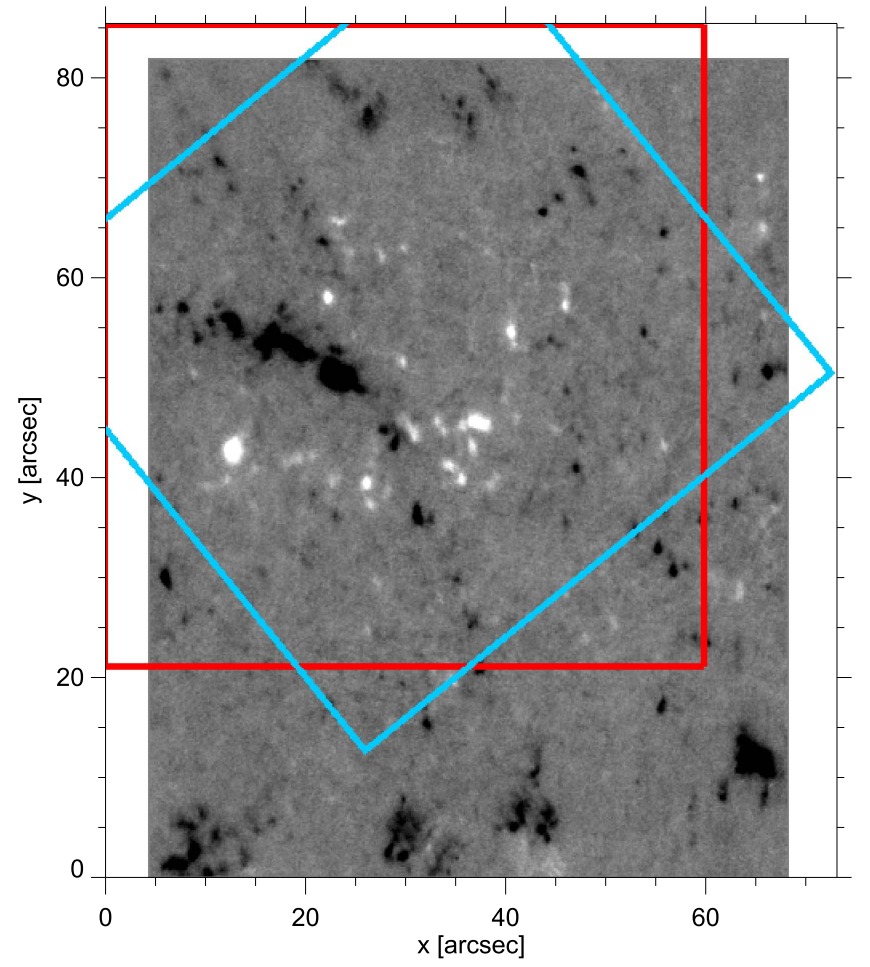

Figure 3. Maximum overlapping of the IRIS slit-jaw (red box) and the SST (blue box) FOVs at the start of the IRIS observations on 2014 May 16 at 07:58:54 UT. The background image is a circular polarization map in $\mathrm{Mg} \mathrm{I} b_{2}$ $5173 \AA$ obtained by the Hinode/NFI at 08:00:34 UT.

Magnetic features that appear and disappear in situ have to be visible in at least two frames. We corrected the YAFTA tracking results using the code developed by Gošić (2015), which resolves the errors made by YAFTA during the tracking process. This code allows us to properly interpret interactions between flux elements and intrinsic changes in the element signals.

\subsubsection{Separation of IN from NE Magnetic Elements}

In order to distinguish between IN and NE flux patches, we have applied the same method as described in Gošić et al. (2014). In this way, the IN regions are considered to coincide with the interior of supergranular cells while the space beyond the IN is the NE regions. Supergranular cells are determined by applying the local correlation tracking (LCT) technique (November \& Simon 1998) to SST Fe $6173 \AA$ A Dopplergram maps, considering the entire time sequence of $3 \mathrm{hr}$. The LCT algorithm calculated horizontal velocities employing a Gaussian tracking window of FWHM 8.5 arcsec, which is large enough to suppress small convective patterns, such are granular flows, while preserving the large-scale supergranular motions. Figure 4 shows separated NE and IN regions, with the red shaded area representing the NE.

Magnetic elements that were in the interior of supergranular cells at the beginning, and those that appeared inside supergranular cells during the observational period, were taken to be IN elements. All other magnetic patches were considered to be NE structures.

\subsection{Cancellation Events}

The cancellation of opposite-polarity flux patches in the photospheric magnetograms lead to the slow fading of the

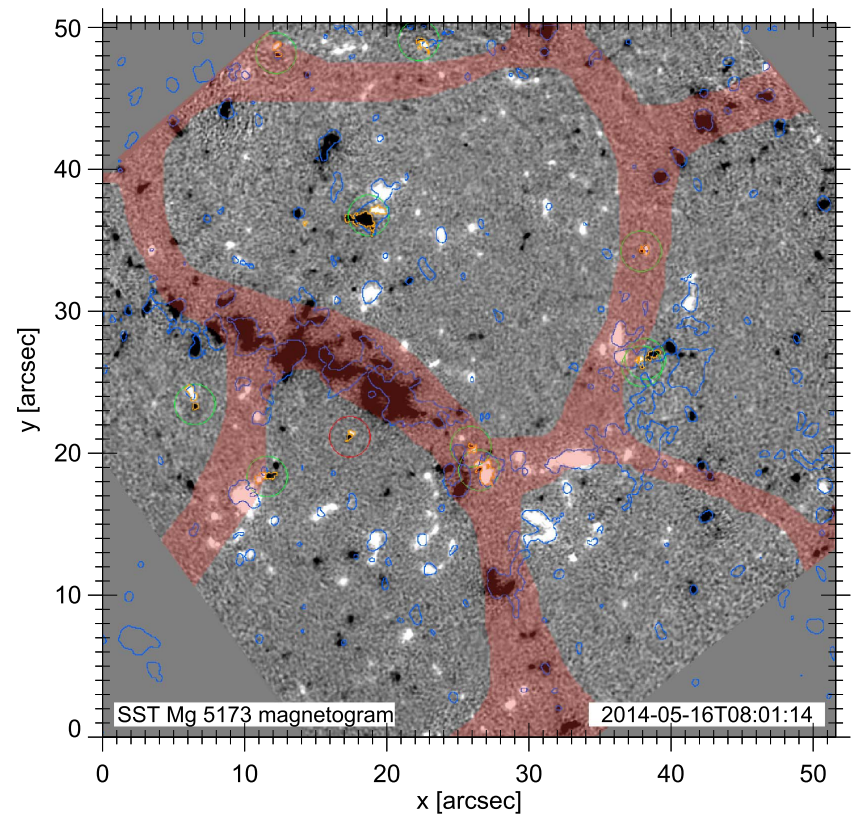

Figure 4. NE and IN regions as determined by the LCT technique applied to SST Fe $6173 \AA$ A Dopplergram maps. The red shaded area coincides with the NE while the non-shaded zones represent the IN, i.e., the interior of supergranular cells.

patches involved in the interaction. Therefore, YAFTA does not see the difference between cancellation and in situ disappearance. For this reason, to detect cancellations, we follow the same strategy as described in detail in Gošić et al. (2014). To summarize, we use the YAFTA output and search for all IN elements that disappear in situ in the current frame. The borders of these elements are dilated by four pixels and those that overlap opposite-polarity patches are identified as canceling features. Each detected pair of canceling elements form a cancellation event to which we assign a unique label. Using these labels, we can study the history of individual cancellation events.

In addition, magnetic elements may undergo partial cancellations as well, which also need to be identified. The reason is that, although flux patches do not disappear through these processes, it is possible that reconnection occurs and releases magnetic energy. Partial cancellations are detected similarly to the previous case. We first expand the borders of each IN element that does not disappear in a given frame by four pixels. If a given element overlaps with an oppositepolarity patch, we inspect whether they are in contact because they form a newly emerging magnetic loop. The latter is done by checking when and how flux patches appear and determine in what direction they are moving. Those patches that appear in situ, that have been visible in the magnetograms in less than five frames, and then move in opposite directions are considered to be bipolar structures and discarded from further study. Otherwise, they are taken as flux features involved in a partial cancellation.

\subsection{Association of IN Cancellations with Bright Grains in SJI 1400}

The cancellation of IN fields is believed to be associated with transient brightenings in the chromosphere and/or transition region. In such cases, we expect to detect signal in SJI 1400 

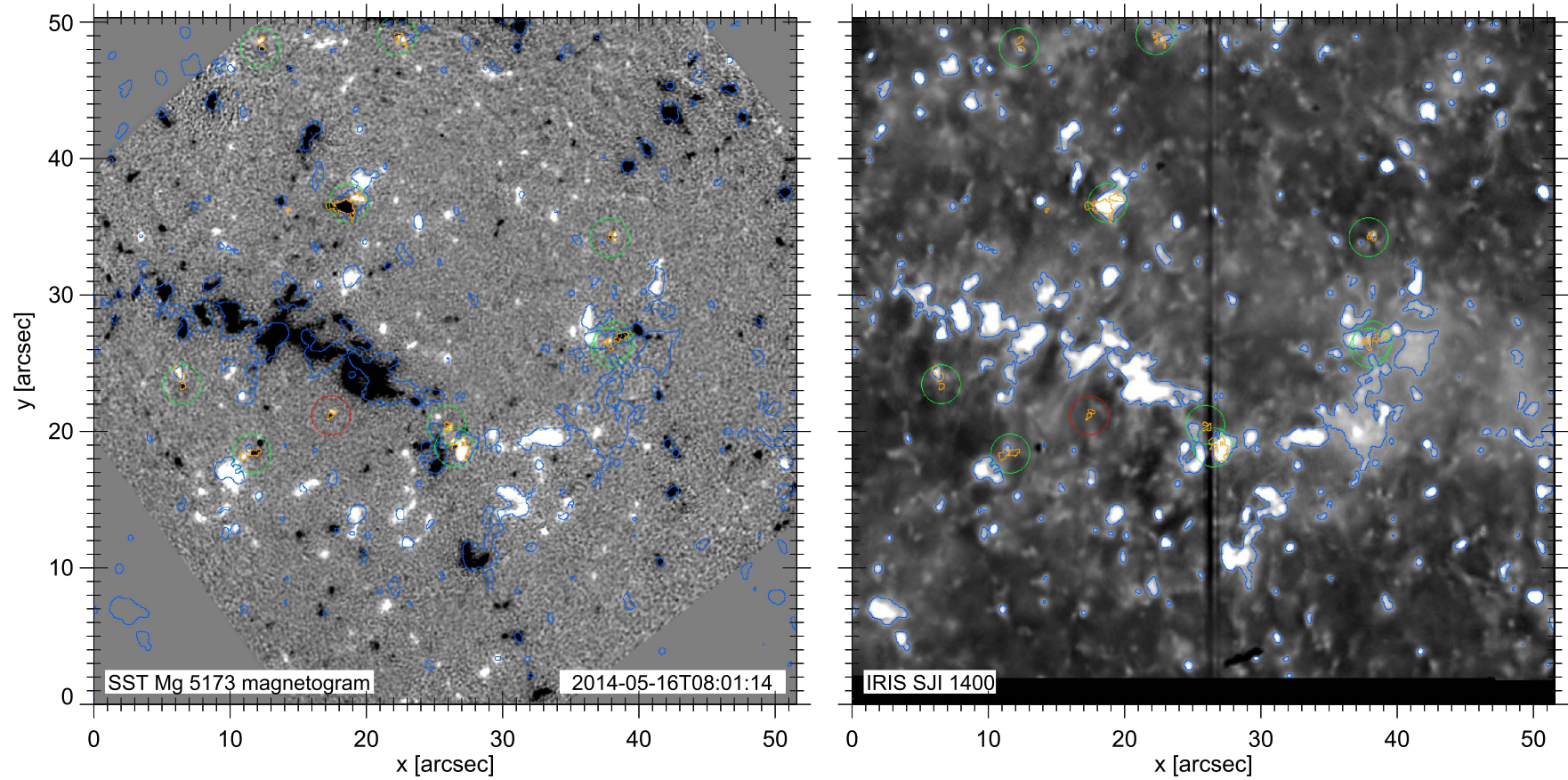

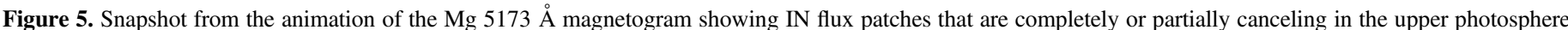

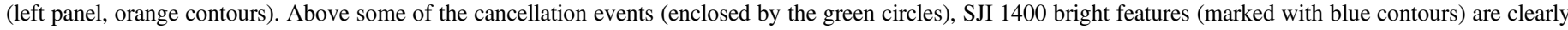

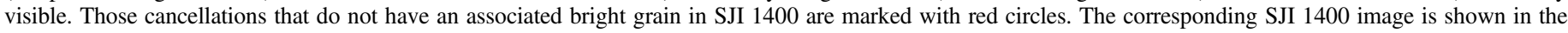
right panel. The animation runs from 07:59 to 10:32 UT on 2014 May 16.

(An animation of this figure is available.)

above cancellation sites, which may be a signature of reconnection processes that release energy and/or hot plasma.

However, special care must be taken with the detection of SJI 1400 bright structures, because not all of them originate from the reconnection of magnetic field lines. Sometimes, bright grains come from the continuum intensity in the SJI $1400 \AA$ filters. They are normally formed in the chromosphere, due to upward propagating acoustic waves in a non-magnetic environment and sometimes they can even reach the transition region (Martínez-Sykora et al. 2015). These shock-related grains are highly transient features. They move fast and have lifetimes of roughly two minutes. Hence, the grains can be removed from the IRIS 1400 SJI images by applying a subsonic filter (Title et al. 1989; Straus et al. 1992) with $5 \mathrm{~km} \mathrm{~s}^{-1}$ as the cutoff. This makes the association of IN cancellations with brightenings in the transition region more reliable.

To check which IN cancellations are interrelated to SJI 1400 brightenings, we first identify these brightenings using YAFTA and the downhill method, considering all of the pixels in SJI 1400 with signal above a threshold level of 60 counts per pixel. We decided to use these settings after careful visual inspection. We have concluded that with such a setup, we do not miss features that we believe are real, i.e., they are persistent and clearly distinguishable from the background noise. We will discuss in Section 4 how different parameters for the detection of SJI 1400 features affect the results. After identification is done, we find the intersections of the canceling magnetic elements detected in the magnetograms by dilating their borders by four pixels. This is done for each cancellation event and in each frame. When an intersection overlaps with any of the detected patches in SJI 1400, we mark the corresponding cancellation event as the one associated with brightenings in the chromosphere/transition region.
The quality of the final tracking and the association of cancellation events with the SJI 1400 signal can be evaluated from the animation of Figure 5. It shows the cancellations detected in the Mg $5173 \AA$ A magnetograms (left panel). Flux patches undergoing cancellations are shown with orange contours during the process; otherwise, their borders are not drawn. If cancellation events have an associated signal in SJI 1400, they are outlined with green circles, and with red if not. Blue contours enclose SJI 1400 pixels where the signal is above 60 counts per pixel (as shown in the right panel).

\subsection{Inversions of IRIS Data}

IRIS provides unprecedented time sequences of $\mathrm{Mg}$ II spectra at high spatial, spectral, and temporal resolution. These spectra represent the most promising IRIS chromospheric diagnostics, permitting us to infer thermodynamical properties of the solar atmosphere (Leenaarts et al. 2013b; Pereira et al. 2015). We used STiC (de la Cruz Rodríguez et al. 2016) to perform chromospheric data inversions. STiC is a new inversion code based on the RH synthesis code (Uitenbroek 2001), and it allows spectral lines in non-local thermodynamic equilibrium (non-LTE), including the effect of partial redistribution in angle and frequency of scattered photons, to be modeled.

We apply the STiC code to our IRIS Mg II spectra in order to retrieve temperature, line-of-sight velocities $\left(v_{\text {los }}\right)$, microturbulence, gas density, gas pressure, and electron density as functions of optical/geometrical depth at IN cancellation sites. These physical parameters will be used to examine the chromospheric response to IN cancellations. For this purpose, we selected every third pixel along the slit at each third time step, and run the inversions in intensity-only mode. This way we created a relatively coarse map of the atmospheric parameters of the QS scanned by the slit. The inversions were carried out in two 

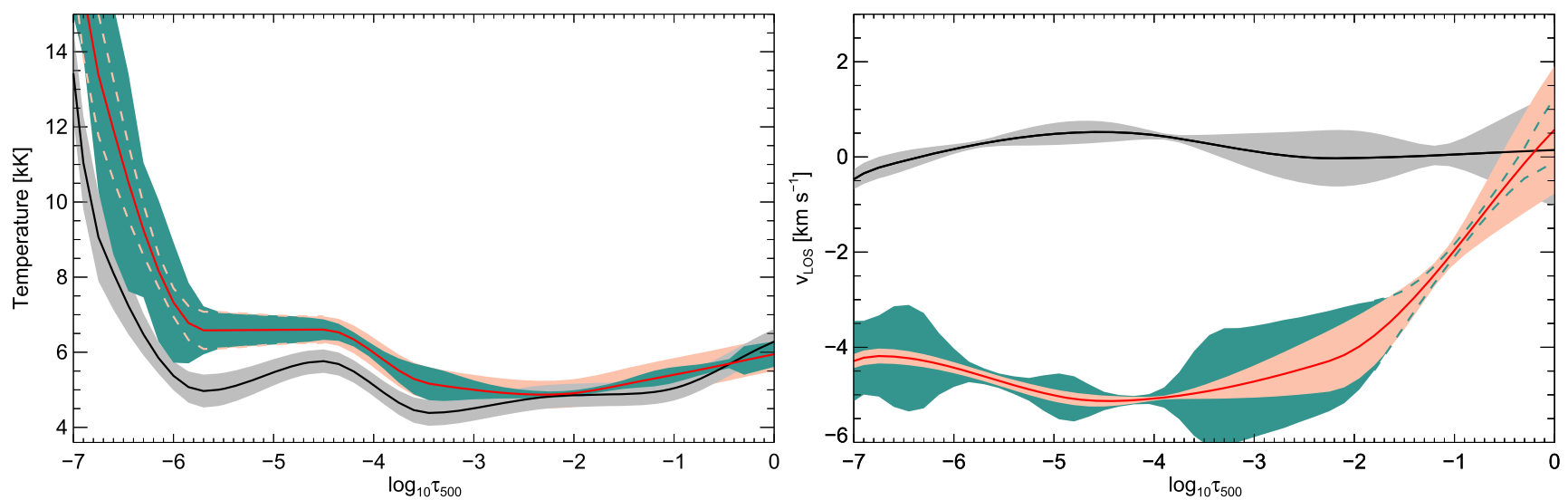

Figure 6. Uncertainties inferred from the inversions of the Mg II $h$ and $k$ and UV triplet lines. The solid black (a QS pixel) and red (pixel within a cancellation region) curves show the temperature (left panel) and LOS velocity (right panel) distributions. The gray and red shaded areas cover their respective uncertainty regions. In addition to this, the green shaded areas around the curves representing the canceling pixel show the uncertainties derived from the corresponding response functions.

cycles, ending with nine nodes in temperature, five nodes in $v_{\text {los }}$, and three nodes in microturbulence. Through trial and error we found that these are the minimum numbers of nodes that provide reasonably good fits to the IRIS QS Mg II $h$ and $k$ and UV triplet lines. The gas pressure at the upper boundary of the model atmosphere is also considered as a free parameter. For the initial atmosphere, we used the FALC model (Fontenla et al. 1993). The initial model contains a given stratification of temperature, $v_{\text {los }}$, microturbulence, and the gas pressure as a function of optical depth. To get sufficient emission in the line cores, we increased the FALC gas pressure by an order of magnitude. The second cycle is initialized by employing the model atmosphere obtained in the first cycle. To derive a more reliable photospheric $v_{\text {los }}$, we included the photospheric Ni I $2814.350 \AA$ line following the strategy from de la Cruz Rodríguez et al. (2016), which is modeled in LTE.

The uncertainties in temperature and LOS velocity that can be expected from the inversions are shown as the gray and red shaded areas in Figure 6. They are computed as the standard deviations of the corresponding atmospheric parameters obtained through inversions of the selected IRIS lines in two pixels using 1000 randomly perturbed initial model atmospheres. One pixel is located within a non-magnetic QS region (black lines) while the other one represents a cancellation region (red lines).

Another way to calculate the uncertainties of the model is given by Equation (42) in del Toro Iniesta \& Ruiz Cobo (2016):

$$
\sigma_{p}^{2} \simeq \frac{2}{n m+r} \frac{\sum_{s=0}^{3} \sum_{i=1}^{q}\left[I_{s}^{\mathrm{syn}}\left(\lambda_{i}\right)-I_{s}^{\mathrm{obs}}\left(\lambda_{i}\right)\right]^{2} w_{s, i}^{2}}{\sum_{s=0}^{3} \sum_{i=1}^{q} R_{p, s}^{2}\left(\lambda_{i}\right) w_{s, i}^{2}},
$$

where $n$ is the number of optical depth points, $m$ is the number of physical quantities varying with depth, while $r$ represents those that are constant with height. $s$ and $i$ scan over the four Stokes parameters and $q$ wavelength samples, respectively. $I_{s}^{\mathrm{syn}}$ and $I_{s}^{\text {obs }}$ are the synthetic and the observed intensity profiles. $R_{p, s}$ are the response functions of a given Stokes parameter to perturbations of the atmospheric quantity $p$, which runs from 1 to $n m+r$. Finally, $\omega_{s, i}$ stands for the weights of the Stokes parameters. The uncertainties are shown in Figure 6 as the green shaded areas around the curves representing the canceling pixel, and for clarity, they are omitted for the QS pixel. As can be seen, they are of the same order of magnitude as the uncertainties derived from the Monte Carlo approach.

\section{Results}

Using the corrected YAFTA output, we identified all total and partial cancellations of flux patches in $\mathrm{Mg} 5173 \AA$ magnetograms. In total, we detected 411 cancellation events (592 magnetic elements), 32 of which include only NE patches. However, since NE cancellations play only a minor role in our observations and for the sake of simplicity, we will consider all cancellation events as IN cancellations further in our analyses. This accounts for 0.1 elements per $\operatorname{arcsec}^{2}$ and per hour. Many cancellations show the standard evolutionary pattern. Two opposite-polarity patches approach each other and start decreasing in size and flux. If the cancellation is total, they completely disappear from the magnetograms. Otherwise, either one or both elements survive the interaction. The interacting magnetic features can also be seen in intensity filtergrams as two tiny bright structures located in intergranular lanes that move toward each other. This is accompanied by increased brightening in the IRIS slit-jaw and Ca II 8542 A images. Some cancellations undergo much more complex evolution, which includes surface processes such as fragmentations and mergings of flux patches.

If we discard 51 cancellation events that are not covered by IRIS due to decreasing overlapping FOV between the SST and IRIS observations, $76 \%$ of the detected cancellation events are cospatial with SJI 1400 signals. The signal-weighted centers of the bright grains are on average by 0.5 away from the corresponding cancellation site centers (defined as the center of intersections between interacting opposite-polarity flux patches). This small dislocation is likely the result of the alignment uncertainty. As mentioned before, SJI 1400 bright features are detected by taking into account all of the pixels above the threshold level of 60 counts per pixel. When the threshold and minimum size of SJI 1400 features are set to 70 counts and 4 pixels, respectively, $70 \%$ of the cancellation events overlap with SJI 1400 structures. This ratio increases to $87 \%$ if all pixels above 40 counts are considered. Too restrictive selection criteria imply that many real signals will remain undetected. On the other hand, too low thresholds yield numerous identifications of spurious features. Since the ratio between these two limiting scenarios does not change by more than $10 \%$, we are confident that the SJI 1400 signal we detect 

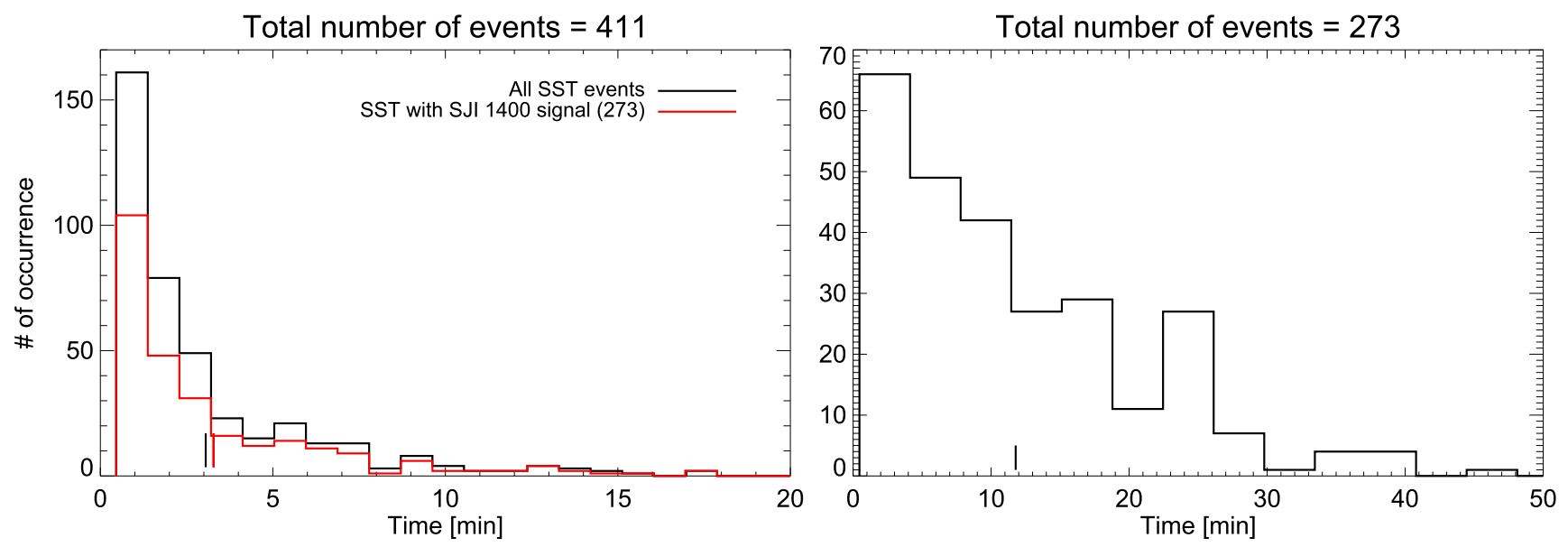

Figure 7. Left panel: lifetimes of all cancellation events detected in the $\mathrm{Mg} 5173 \AA$ magnetograms (black line) and those that have associated SJI 1400 signal (red line). The bin sizes are $55 \mathrm{~s}$. Right panel: lifetimes of SJI 1400 bright grains assigned to cancellations in Mg 5173 A magnetograms (the bin size is 220 s). The black and red vertical lines mark the mean values of the corresponding lifetime distributions.

above the cancellation regions is real. In $\sim 80 \%$ of cases, this signal starts to form, on average, $\sim 4$ minutes before we detect cancellations in Mg $5173 \AA$ magnetograms.

The events without associated SJI 1400 features may imply several possibilities: they are the result of our selection criteria, SJI 1400 images do not have sufficiently high sensitivity, or canceling flux features are simply too weak to affect the chromosphere and layers above.

Hereafter, we characterize the following parameters of the detected cancellations: lifetime, size, and atmospheric physical parameters (from the inversions). The latter are used to estimate the total energy released through the QS cancellations. A general description of two cancellation events is provided after this characterization. We have proceeded with a detailed analysis of the second event for which we also have IRIS spectra.

\subsection{Lifetime and Size}

The lifetime distributions of the detected cancellation events are displayed in Figure 7 (left panel). In our magnetogram sequences, an event is considered to live from the frame in which flux patches start to interact until they get separated or one of them disappears. The lifetimes of cancellations range from 1 minute up to 22 minutes. The lower limit is set by the cadence of the SST observations. Both distributions, for all cancellation events (black line) and for those with associated SJI 1400 bright features (red line), have practically the same mean lifetime of about 3 minutes.

However, SJI 1400 bright structures above cancellation events live 11.8 minutes on average (right panel in Figure 7), i.e., much longer than the cancellations seen in $\mathrm{Mg} 5173 \AA$ magnetograms. We note that the downhill method (Welsch \& Longcope 2003), used to identify SJI 1400 bright grains, detects local maxima and classifies each of them as a distinct patch. This approach is suitable whenever it is necessary to separate individual patches from large complex structures. However, this means that the lifetimes of SJI 1400 bright grains associated with cancellation events are determined by the downhill method itself, keeping in mind its limitations (DeForest et al. 2007).

The size distribution of the detected SJI 1400 bright grains is shown in Figure 8. The lower and upper limits of the

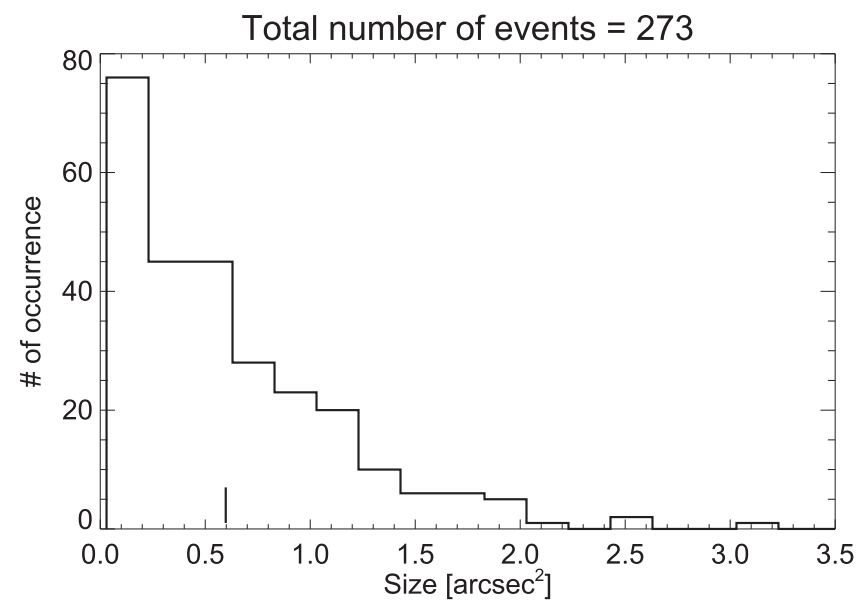

Figure 8. Size distribution of IRIS SJI 1400 bright grains. The bin sizes used are $0.2 \operatorname{arcsec}^{2}$. The black vertical line indicates the mean value of the distribution.

distribution are $0.03 \operatorname{arcsec}^{2}$ and $3.4 \operatorname{arcsec}^{2}$, respectively. The mean size of bright grains is about $0.6 \operatorname{arcsec}^{2}$. Interestingly, this is similar to the mean size of IN flux concentrations measured in Hinode/NFI photospheric magnetograms (Gošić 2015).

\subsection{Examples of Canceling Events}

In this section, we will describe in detail the evolution of two cancellation events. They show how opposite-polarity IN magnetic fields interact, affecting the upper solar atmosphere up to the transition region.

\subsubsection{Example 1}

Example 1 portrays a simple canceling scenario of two small IN flux concentrations. The time evolution for this example is displayed in Figure 9 and the accompanying movie, where the flux cancellation region is enclosed by a red contour. The figure shows 12 observables, from left to right: continuum intensity at Fe $6173 \AA$ linear polarization and lower photospheric magnetograms taken in the same line $( \pm 56 \mathrm{m \AA})$, wing intensity

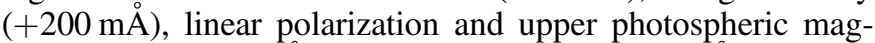
netograms $( \pm 200 \mathrm{m \AA})$ recorded in the $\mathrm{Mg} 5173 \AA$ line, and 


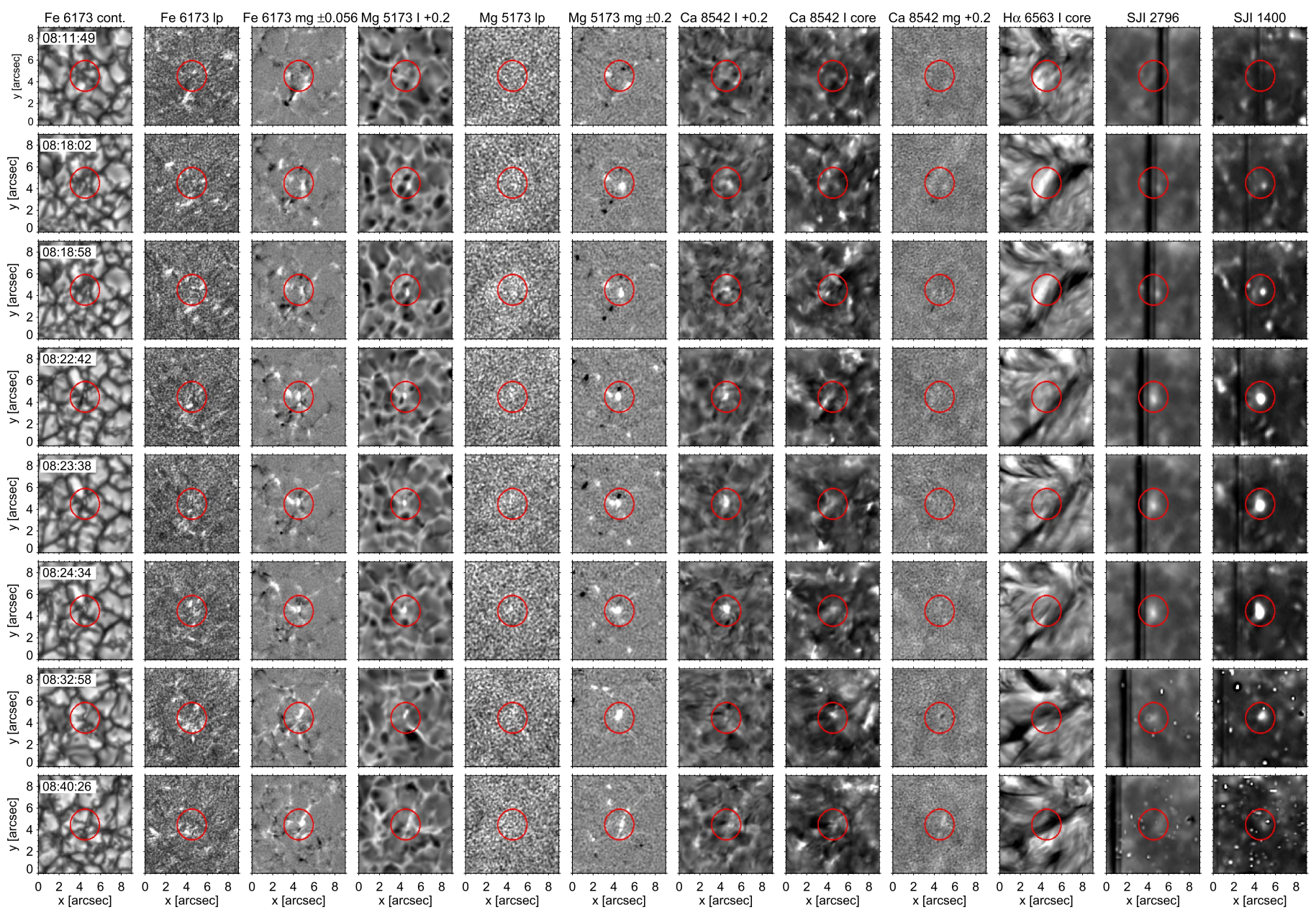

Figure 9. Temporal sequences of several SST and IRIS observables during the flux cancellation phase. From left to right: continuum intensity, linear polarization and magnetograms in Fe I $6173 \AA$, intensity images in the red wing of the $\mathrm{Mg} 5173 \AA$ line and the corresponding linear polarization maps and magnetograms, filtergrams in Ca II $8542 \AA$ (the red wing and core), and Ca II magnetograms, and the last three rows show the core in H $\alpha 6563 \AA$ and IRIS SJI 2796 and 1400 filtergrams. The red circles outline two canceling IN flux concentrations located around $(x, y)=\left(5^{\prime \prime}, 5^{\prime \prime}\right)$. Starting from 08:18:02 UT, the filtergrams showing the chromosphere and transition region reveal brightening features at the observed location. IN flux concentrations in the lower-lying photosphere (as seen in the photospheric magnetograms) are seen to be canceling. The animation runs from 07:59 to 8:30 UT on 2014 May 16.

(An animation of this figure is available.)

intensity maps in the wing $(+200 \mathrm{m \AA})$ and core of Ca II 8542 where brightenings are more pronounced. The final four rows are Ca II magnetograms, intensity filtergrams in the core of $\mathrm{H} \alpha 6563 \AA$ and IRIS 2796 and 1400 slit-jaw images. The associated movie shows the same observables. These temporal sequences clearly show the cancellation of IN fluxes and the chromospheric response to it.

The first sign of chromospheric activity above the cancellation region appears at 8:11:49 UT in the red wing of Ca II $8542 \AA$. We see a small, faint brightening at $(x, y)=(5 . " 5$, 3. .9), probably triggered by the cancellation of the very small negative flux patch with the stronger positive flux concentration visible in Fe magnetograms. None of the other filtergrams show any activity at that location; neither do $\mathrm{Mg}$ magnetograms show interaction of flux patches. Three minutes later, a small bright structure appears in IRIS SJI 1400, coinciding with the positive magnetic element at $(x, y)=\left(5{ }^{\prime \prime} 5,3\right.$ "'9). It becomes stronger and larger very fast while the signal at Ca II $8542+$ $0.2 \AA$ follows a similar evolutionary pattern.

In the meantime, two negative flux patches visible in the $\mathrm{Mg}$ $5173 \pm 0.2 \AA$ magnetograms at $(x, y)=\left(5{ }^{\prime \prime} 7,5\right.$ " 5$)$ are being dragged by granular motions within intergranular lanes and coalesce into a larger, stronger individual flux structure at 8:20:50 UT. The newly created flux concentration migrates toward the positive element $(4 ! " 5,4$ !" 5$)$. The evolution of their corresponding footpoints can also be seen in the first and fourth rows in Figure 9 as small-scale bright points (BPs) between granular cells. These BPs can be better discerned in the intensity maps in the red wing $(+200 \mathrm{~m} \AA)$ of the $\mathrm{Mg} 5173 \AA$ line, especially the one corresponding to the positive-polarity element. They also coincide with the strong local downflows visible in the $\mathrm{Fe}$ and $\mathrm{Mg}$ Dopplergrams. Simultaneously, the first hint of a somewhat permanent brightening shows up in the core of the Ca II line.

The cancellation in both $\mathrm{Fe} 6173 \pm 0.056 \AA$ and $\mathrm{Mg} 5173 \pm$ $0.2 \AA$ magnetograms starts to be visible from approximately 08:21:46 UT. By that time, intensity enhancements are easily discernible in the Ca II $8542+0.2 \AA$ and IRIS slit-jaw images. They attain their maximum in size and brightness from 08:23:38 UT to $08: 25: 12$ UT when an apparent bright structure is visible in both Ca II filtergrams which can be interpreted as a signature of upflowing plasma (see below). During this period, there is a weak 
linear polarization (LP) signal in the blue wing of the Mg $5173 \AA$ line. Since the signal is too weak, it can easily be the result of intensity to Stokes $Q$ and $U$ crosstalk or noise fluctuations.

The negative flux patch disappears completely from the $\mathrm{Mg}$ magnetograms at 08:25:30 UT. The positive patch, however, survives the interaction and continues its evolution. In $\mathrm{Fe}$ magnetograms, we can still follow the flux cancellation at the photospheric level. The disappearance of the negative flux structure is followed by strong LP located at $(x, y)=(\sim 4 ! .5$, $\sim 5$.' 5), between positive and negative patches. It is possible that the detected LP feature is a signature of a newly created descending loop. In the meantime, filtergrams in the core of the $\mathrm{H} \alpha 6563 \AA$ line reveal the formation of a dark cloud at $8: 24: 34$ UT, $(x, y)=(\sim 5 ! 7, \sim 5 !$ ! 5). The cloud transforms into an elongated dark structure and can be observed until 08:35:09 UT, when it merges with another dark fibril coming from its right side. Bright structures in the Ca II, SJI 2796, and SJI 1400 filtergrams can be observed in several more time steps. They first disappear from the Ca II $8542+0.2 \AA$ image at 8:30:10 UT, and from the IRIS slit-jaw images about 3 minutes later. Interestingly, the movie shows that transient brightenings in the core of the Ca II $8542 \AA$ line, above the cancellation event, can be further observed until 08:41:03 UT.

Figure 10 shows the temporal evolution of the Stokes I profiles in the Ca II $8542 \AA$ line. The black solid curves in the left panels represent $\mathrm{Ca}$ II intensity spectra obtained at the position marked with the red cross in the filtergrams in the right column. The dotted green curves show the average QS profile calculated in the available SST FOV, avoiding the strongest, negative network structure. Time is shown in the lower-left corners and increases from top to bottom. The vertical dotted green lines in the left column mark the wavelength of the resting line center. The Ca II $8542+0.2 \AA$ wavelength is shown with a red dotted vertical line, and the corresponding filtergrams are displayed in the right column.

The first spectral line is taken at 08:11:49 UT, just before the appearance of strong bright structures in the Ca II filtergrams. As cancellation unfolds with time, the spectral profiles in the cancellation region start to be distinctively different from the QS average. The line center clearly shifts toward the blue side of the line while developing strong asymmetry. There is a very sharp gradient on the red side and a much more extended blue side. The line intensity shows enhancement both in the wings and core during the cancellation. In addition to this, at 0.3 and $0.4 \AA$, the line clearly turns into emission, which is not present in the blue wing. We will discuss in Section 5 a possible mechanism that may be responsible for producing the observed Ca II $8542 \AA$ Stokes I profiles.

\subsubsection{Example 2}

Example 2 is more involved but provides essential information on the cancellation process through available IRIS spectra. This example is displayed in Figure 11 and shows interaction between two negative and two positive flux patches, as detected in $\mathrm{Mg}$ magnetograms. The temporal evolution of this cancellation event and the atmospheric layers above can also be seen in the accompanying movie. It shows the same observables as in the case of example 1 .

From the beginning of the temporal subsequence, the SST continuum intensity maps expose bright points coinciding with the magnetic elements involved in the cancellation (enclosed by the red circles). At the same time, the negative-polarity patches

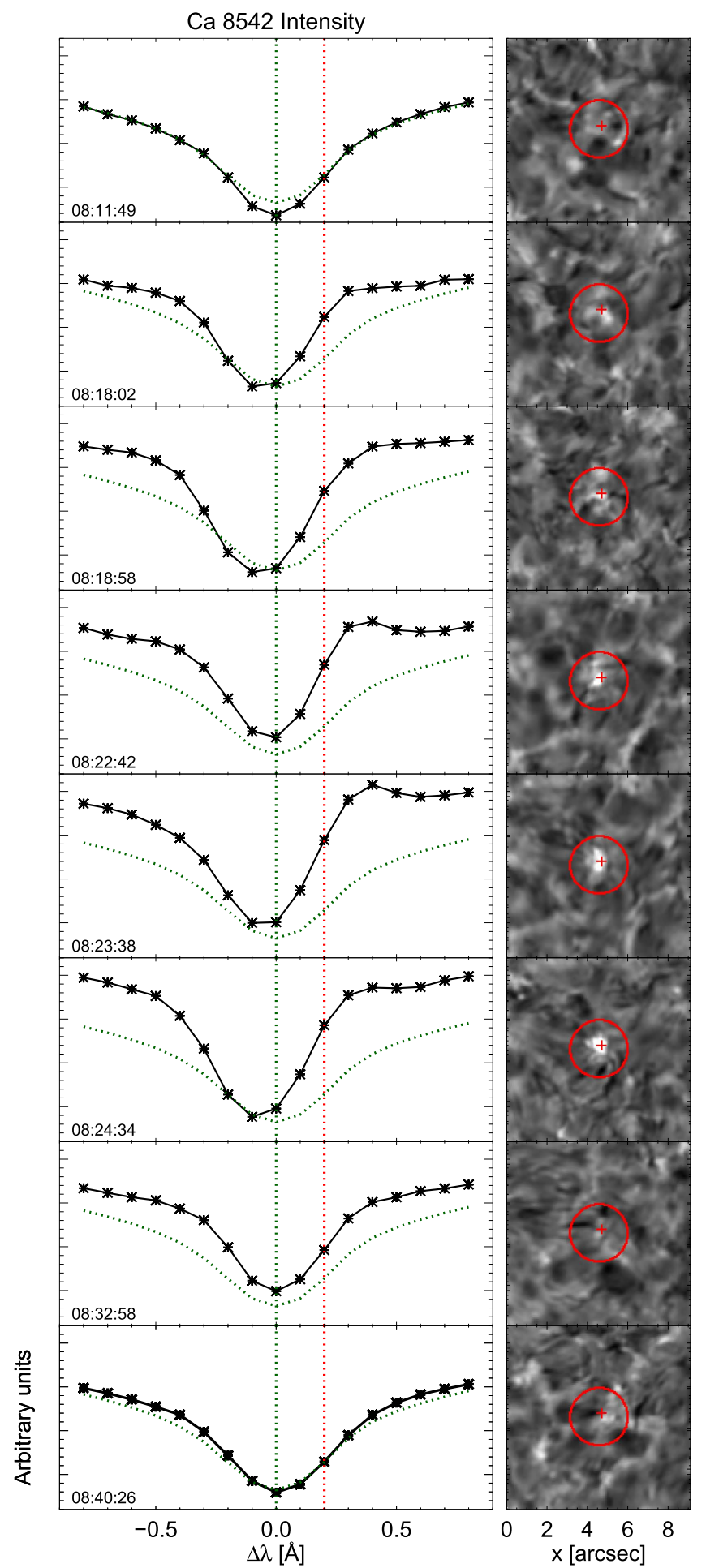

Figure 10. Temporal evolution of the Stokes $I$ profiles in the Ca II $8542 \AA$ line (black lines) formed at the center of the cancellation event presented in Figure 9. The spectra are observed at the location marked with the red cross in the filtergrams shown on the right. The dotted green curve represents the average QS profile in the accessible SST FOV. Time increases from top to bottom, as indicated in the lower-left corner of each panel. The vertical dotted green and red lines in the spectra panels represent the rest wavelength of the line center (i.e., $8542 \AA$ ) and the one at which the intensity maps in the right column are taken $(8542+0.2 \AA)$, respectively.

are cospatial with localized brightenings, clearly visible in filtergrams taken in the wing and core of Ca II $8542 \AA$. These brightenings appeared as soon as the positive flux patch at 

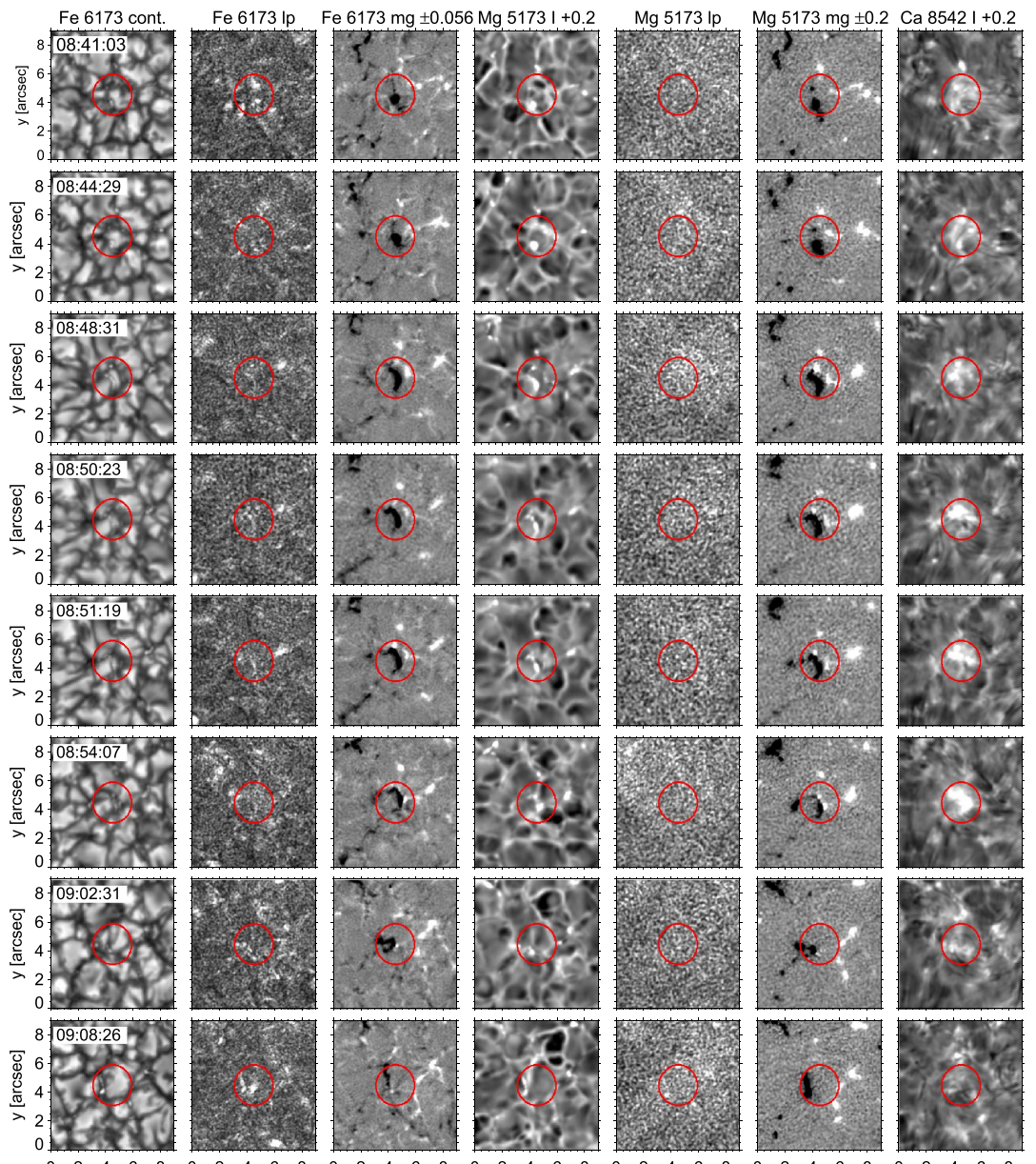

$\begin{array}{lll}2 & 4 & 6 \\ x & {[\operatorname{arcsec}]}\end{array}$
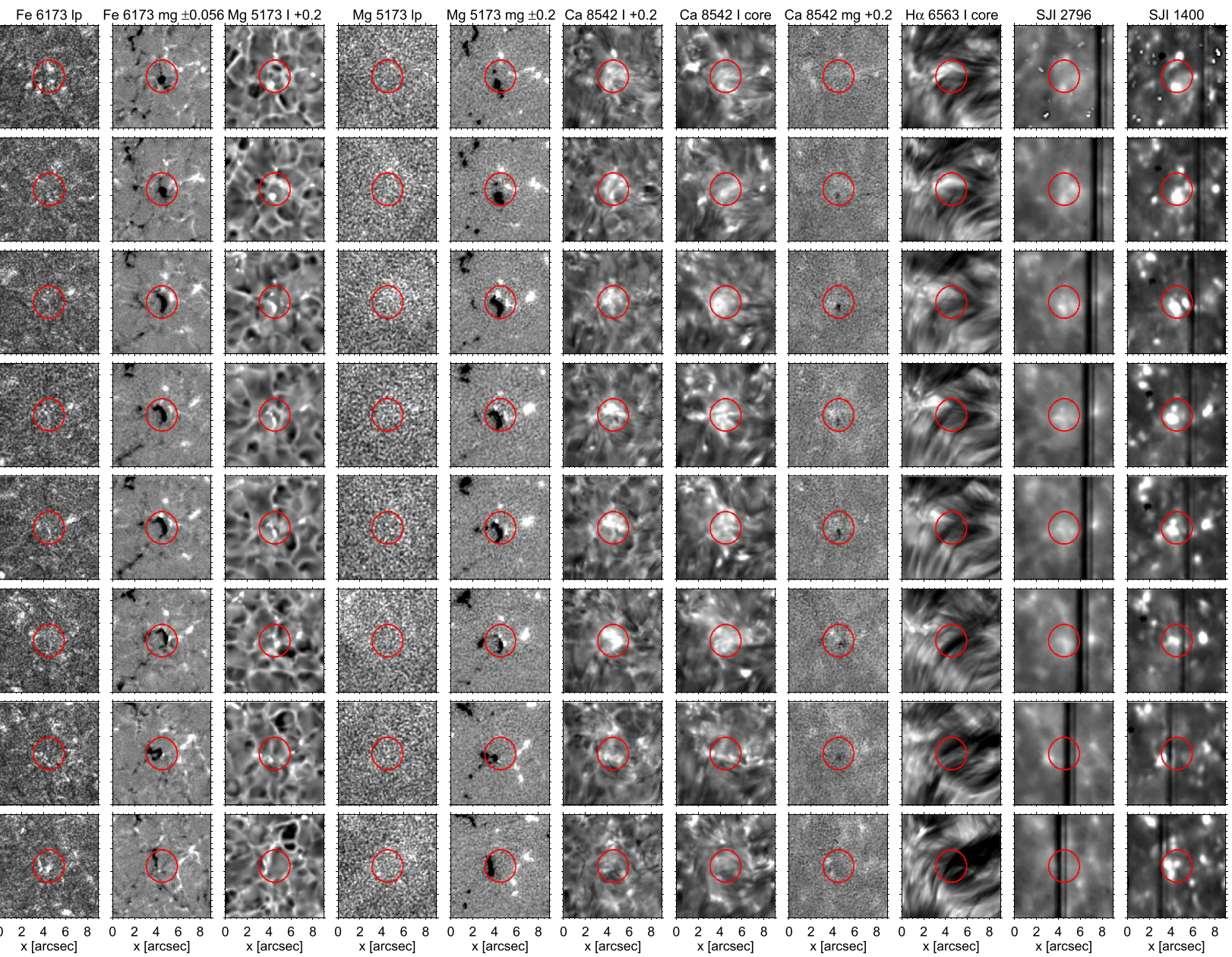

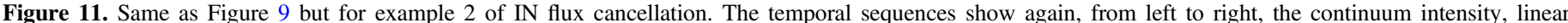

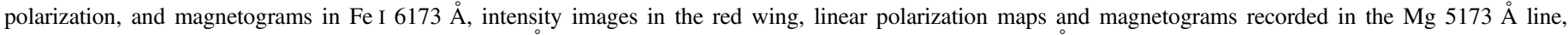

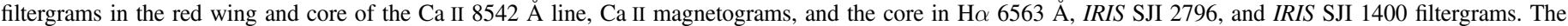

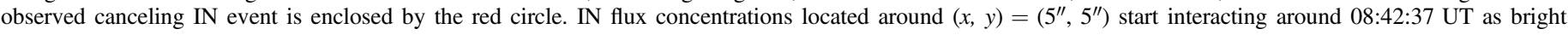

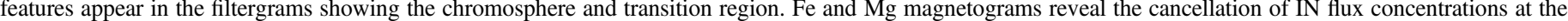
photospheric level. The animation runs from 08:41 to 9:12 UT on 2014 May 16.

(An animation of this figure is available.)

$(x, y)=\left(4{ }^{\prime \prime} 5,6\right.$ "' 5$)$ approached the negative patches (this spatiotemporal evolution is not shown here). On the other hand, just like in the first example, there is a noticeable chromospheric activity between positive and negative flux concentrations appearing at 8:42:37 UT in the wing of Ca II $8542 \AA$ A. Small bright patches appear above the positive magnetic elements at $(x, y)=\left(5 \prime \prime 5,5^{\prime \prime}\right)$. This may indicate the onset of reconnection where associated currents are causing heating and produce brightenings along the field lines connecting the footpoints. In the next frame, a bright feature shows up in the core of the Ca II $8542 \AA$ line and is located between positive and negative flux structures. Only one minute later, at 8:43:51 UT, IRIS SJI 1400 filtergrams reveal signal intensification in the same region.

Magnetic flux cancellation is detected for the first time in $\mathrm{Mg}$ magnetograms at 8:44:47 UT when two small opposite-polarity elements start interacting $\left(4 "{ }^{\prime \prime} 5,5^{\prime \prime}\right)$. Meanwhile, Ca II filtergrams show the evolution of bright features above the cancellation site. They seem to take the form of loop-like structures, oriented in such a way as to indicate the connection between interacting magnetic elements.

The other two opposite-polarity flux patches start canceling at 8:47:35 UT, as another roundish bright feature develops in
IRIS SJI 1400, just left of the slit. While cancellation unfolds in the photosphere, $\mathrm{H} \alpha$ measurements show the formation of dark fibrils at $(x, y)=\left(4^{\prime \prime}, 5^{\prime \prime}\right)$. By 8:56:55 UT, the smaller positive flux patch completely disappeared. During this period, the signal in the wing and core of Ca II 8542 A remained strong, showing dynamic bright structures above the cancellation site. The dark fibrils visible in the $\mathrm{H} \alpha$ core grow with time and are oriented perpendicular to the polarity inversion line. Part of the fibrils dissolved around 9:00:39 UT and part got mixed with other overlying $\mathrm{H} \alpha$ features.

At 9:03:27 UT, the second positive-polarity patch disappeared from $\mathrm{Mg}$ magnetograms, but can be observed further in Fe magnetograms, until the end of the temporal sequence. The LP signal is visible only in the Fe $6173 \mathrm{~A}$ line at the end of the movie and is probably not generated by the cancellation event, but is due to the flux emergence in the vicinity, at $(x, y)=(4 ! 5,4 ! " 5)$. Bright features disappeared from the Ca II wing filtergrams at 9:05:56 UT and from the core at 9:07:48 UT.

The left column in Figure 12 displays the temporal evolution of the Stokes $I$ profiles in the Ca II $8542 \AA$ line, formed inside the cancellation region as denoted by the red cross. The 


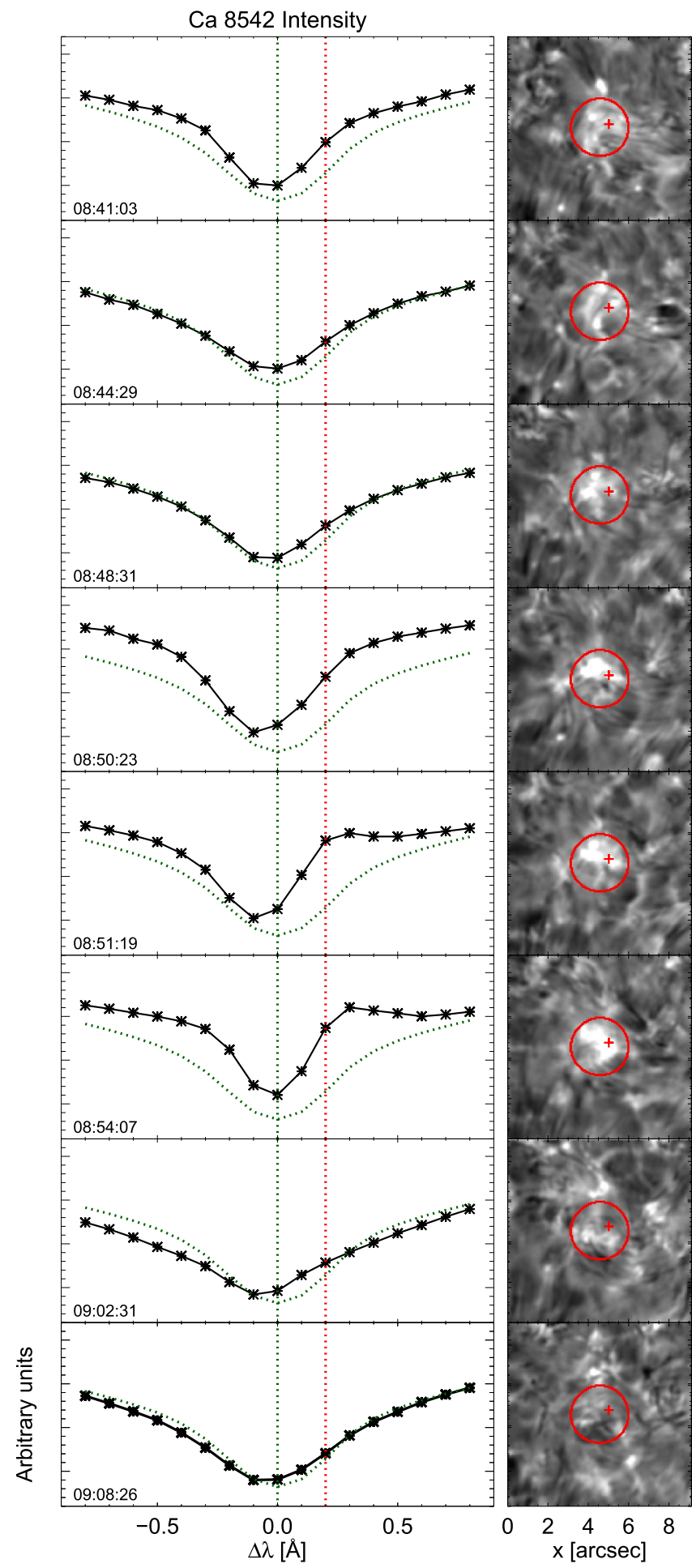

Figure 12. Same as Figure 10 but for example 2. The temporal evolution of the Stokes $I$ profiles in the Ca II $8542 \AA$ line (black lines) shows the line intensity enhancement in the wings and core during the cancellation while the red wing of the Ca II $8542 \AA$ line turns into emission.

corresponding Ca II $8542+0.2 \AA$ filtergrams are given in the right column. The intensity profiles exhibit a very similar temporal evolution to those in example 1. Before and at the beginning of the cancellation process, intensity profiles are similar to that in the average QS, although slightly blueshifted. With time, the Ca II $8542+0.2 \AA$ line exhibits enhancement at all wavelengths and becomes asymmetric with the red wing (from 0.3 to $0.4 \AA$ ), turning into emission around 8:51:19 UT. The profiles are again clearly shifted toward the blue side during cancellation.

Characteristic IRIS spectra for case 2 are given in Figure 13. The black solid line shows the spectra taken at the cancellation site at 8:55:59 UT (marked with the red cross in Figure 12). The non-magnetic QS spectra are shown with the red solid line. The Mg II $h$ and $k$ lines, which are formed above the flux cancellation site, are much stronger and more asymmetric than in the "non-magnetic" QS environment. There is a clear intensity enhancement throughout the $\mathrm{Mg}$ II $k$ and $h$ spectral domain, where the $\mathrm{k} 2 \mathrm{r}$ and $\mathrm{h} 2 \mathrm{r}$ are stronger than $\mathrm{k} 2 \mathrm{v}$ and $\mathrm{h} 2 \mathrm{v}$ peaks. In addition to this, the continuum intensity also shows an increase during the flux cancellation. Such a profile may indicate a substantial amount of energy released through the cancellation process, resulting in the local heating of the upper chromospheric layers. The intensity ratio between the blue and the red peak suggests the possible presence of upflowing plasma (Leenaarts et al. 2013b). Si IV $1400 \AA$, Si IV $1394 \AA$, and C II $1336 \AA$ lines provide additional information on the upper chromospheric/transition region heating. These lines all show small but noticeable intensity increases (black solid lines) compared to the non-magnetic QS profiles that are completely buried in the noise (red solid lines).

In order to decipher whether the Mg II $h$ and $k$ profiles shown in Figure 13 indicate heating of the upper solar atmosphere, we inverted these lines using STiC. The best fit is presented with the blue solid line in the upper panel of Figure 14. The red solid curve shows the observed spectra. The Ni I line used to better constrain the LOS velocities in the photosphere is shown in the inset. Here, the best fit is plotted with a dashed blue curve for the purpose of visualization. The observed profiles are very well reproduced, except for small discrepancies in the core of the Mg II lines. The temperature stratification in the resulting model atmosphere is displayed in the bottom-left panel of Figure 14. It can be seen that the photospheric and chromospheric temperatures inferred from the inversions in the case of cancellation (red line) are higher than in the QS case (black line). The corresponding shaded areas represent their respective uncertainties. Our results suggest that the temperature in the solar atmosphere increases by up to $2000 \mathrm{~K}$ when IN magnetic elements start canceling. The temperature difference between the canceling and non-magnetic QS cases is the highest in the range $\log _{10} \tau_{500}=[-6.5,-4]$. This is expected because the canceling $\mathrm{Mg}$ II profiles normally has much broader wings and significantly stronger $k$ and $h$ peaks, which is mainly determined by the temperature stratification. Further evidence of the temperature increase around the cancellation region is provided in Figure 15 (left panel). The coarse map (every third pixel along the slit per third time step is selected) shows the temperature at $\log _{10} \tau_{500}=-5.85$, where one of the temperature nodes is located (this corresponds to the mid-chromospheric layers in the FALC model). We can see the temperature distribution in the vicinity of the cancellation region. The black contour indicates the borders of the canceling flux patches detected in the photosphere as the slit passes across them. Interestingly, the highest temperatures are found just above the cancellation site. The reliability of the inversions and the reconstructed temperatures can also be assessed by comparing the temperature map inferred from the inversions (left panel) with the map constructed from the average of the IRIS Mg II k2v and $\mathrm{k} 2 \mathrm{r}$ peak intensities. Those $\mathrm{Mg}$ II components are, based on 


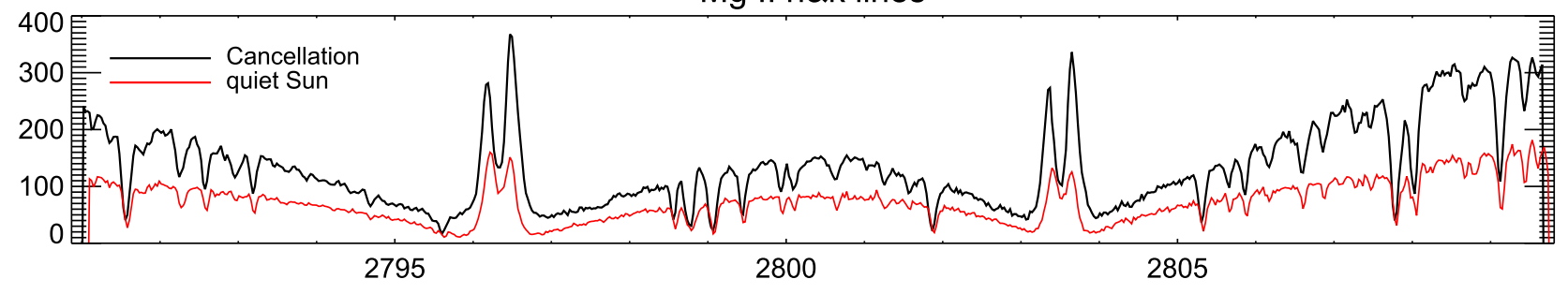

Si IV $1403 \AA$

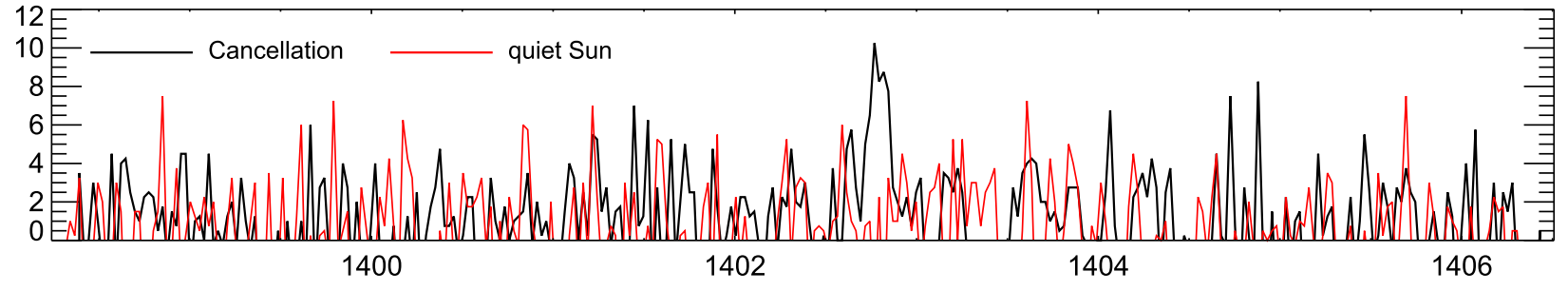

Si IV $1394 \AA$

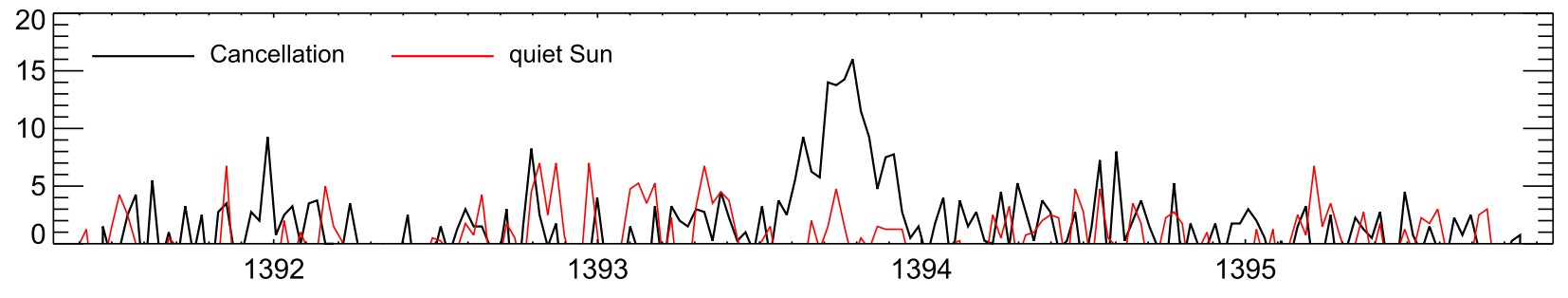

C II $1336 \AA$

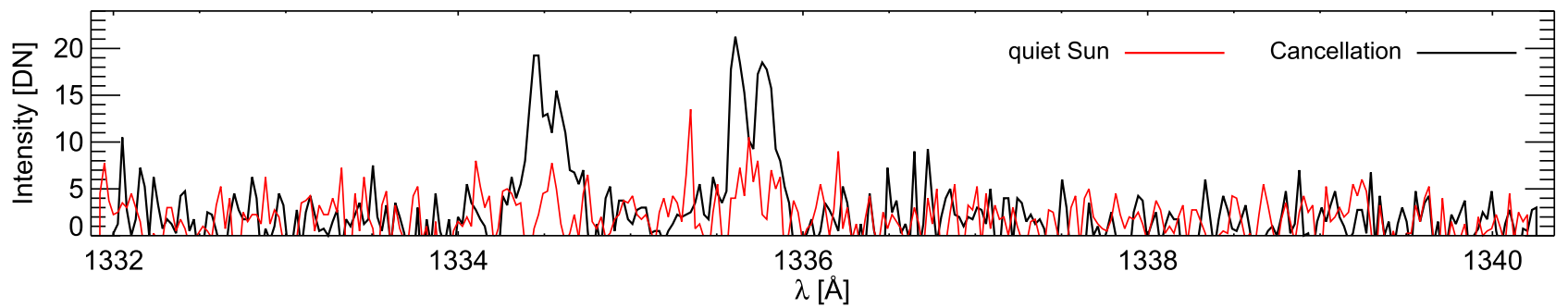

Figure 13. IRIS spectra recorded during the cancellation event presented in Figure 11. Panels, from top to bottom: the IRIS Mg II $h$ and $k$ lines with the surrounding spectral lines and continuum, the Si IV $1400 \AA$ and $1394 \AA$ lines, and C II $1336 \AA$ spectral window. The black solid lines represent the IRIS spectra during the cancellation process, while the red line corresponds to the QS pixel outside of the cancellation region. The spectra are recorded at 8:55:59 UT.

comparisons with advanced numerical simulations, considered to be good indicators of chromospheric temperatures (Pereira et al. 2013). The Pearson linear correlation coefficient for these two maps is 0.81 .

LOS velocities in the canceling and non-magnetic pixels are marked with solid red and black lines in the bottom-right panel of Figure 14, respectively. Again, the shaded areas show their uncertainties. Since the $\mathrm{Mg}$ II lines are insensitive to perturbations of the photospheric LOS velocities, this physical parameter is mostly constrained by the Ni I line and hence less trustworthy at photospheric heights. In any case, the profiles mostly show either zero or positive values (i.e., downward) throughout the lower solar atmosphere. The canceling pixel shows upflows in the upper chromosphere, where IRIS Mg II spectral lines are sensitive and provide more reliable values. On the other hand, $v_{\mathrm{los}}$ in the QS pixel remains around zero. Figure 16 reveals $v_{\mathrm{los}}$ and microturbulence maps above the cancellation site (enclosed by the dark contours) and its surroundings. The panels in the upper row show the $v_{\text {los }}$ derived from the Doppler shifts of the IRIS Mg II $k_{3}$ (left) and $k_{2}$ (right) components following the recipe by Pereira et al. (2013). We can see that these maps display practically the same features although the velocities are higher in the $k_{3}$ map. The $v_{\text {los }}$ reconstructed from inversions is shown in the lower-left panel and despite having smaller amplitudes, it resembles the $k_{2}$, map providing confidence in the inversion results. The Pearson linear correlation coefficient has its highest value of 0.72 at $\log _{10} \tau_{500}=-5.85$. As can be seen, there are strong upflows inside the cancellation region, reaching speeds of up to $-7 \mathrm{~km} \mathrm{~s}^{-1}$ at the height of $\log _{10} \tau_{500}=-5.85$. These results indicate the presence of plasma being pushed up to the chromosphere at an average speed of $-4 \mathrm{~km} \mathrm{~s}^{-1}$. It is reasonable to assume that this plasma, if it stays at the same temperature, should eventually fall down to the surface and downflows would be detected. However, we do not see this effect in our observations because the slit is moving across the 

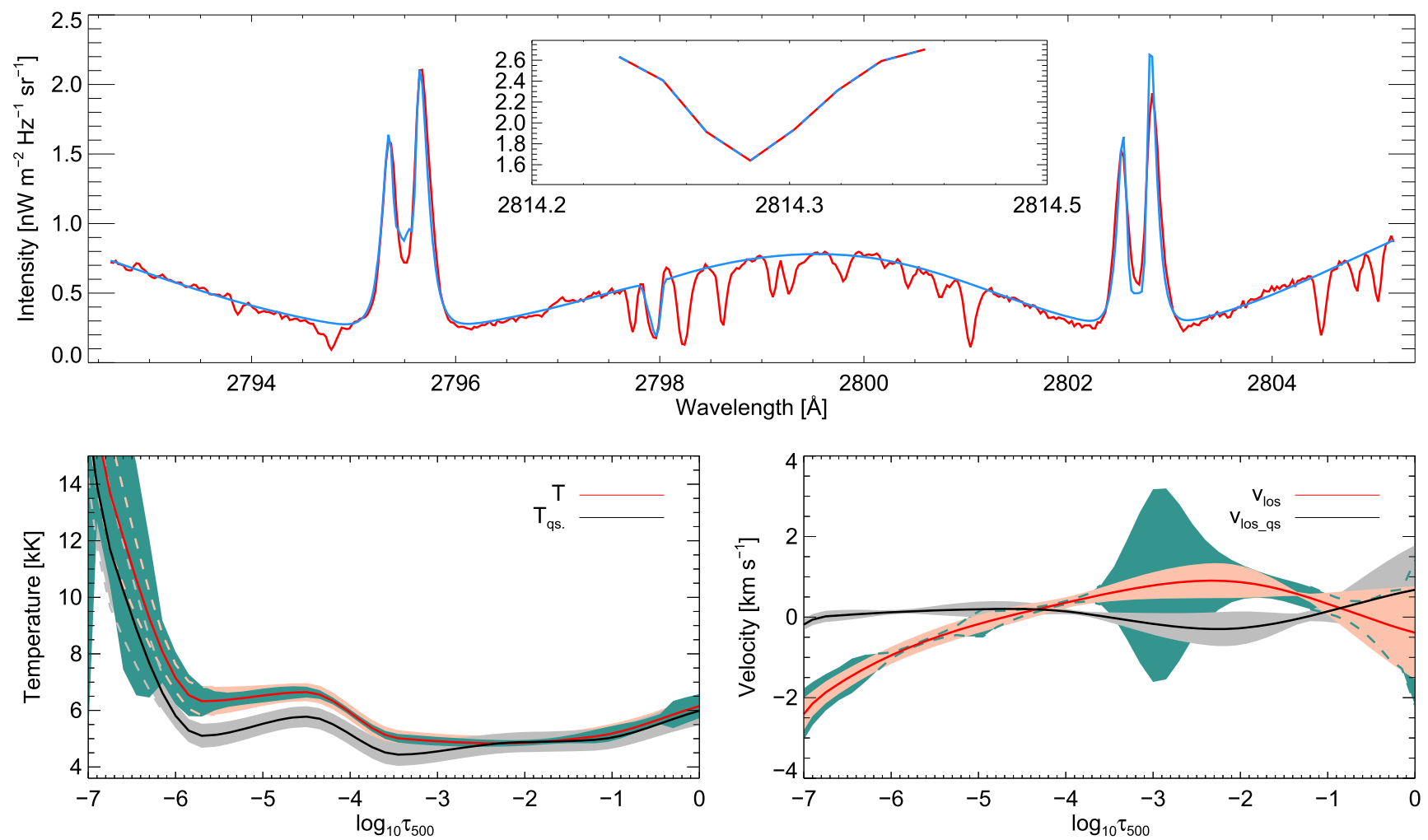

Figure 14. Upper panel: observed Mg II $h$ and $k$ spectra (red) and best fit (blue) for a pixel inside the cancellation region for which the IRIS Mg II $h$ and $k$ and UV triplet lines are inverted. The inset depicts the observed Ni I $2814.350 \AA$ Aine (red) used to better constrain velocities in the photosphere and best fit (dashed blue line). Bottom panels: the retrieved temperature (left) and $v_{\text {los }}$ (right) are indicated with the red curves. Here we use the convention that positive values represent downflowing plasma. As a reference, the black lines show the inverted QS temperature and $v_{\text {los }}$ stratifications. Their respective uncertainties from multiple inversions are shown with the gray and red shaded areas. Those uncertainties calculated from the response functions are plotted with the green shaded areas.

cancellation region and therefore, does not sample the same environment all the time. Still, we see downflows in several pixels closer to the end of the cancellation event, from $\Delta t=23$ minutes until $\Delta t=27$ minutes. The reconnection of magnetic field lines increases the temperature locally, but seems to also cause higher microturbulent velocities in the chromosphere (lower-right panel in Figure 16). The similarity of the microturbulence and temperature maps may also be, to some extent, the result of an interplay between these two atmospheric parameters since the wing widths of the $\mathrm{Mg}$ II lines and the subordinate triplet lines are sensitive to both of them (Carlsson et al. 2015; see also Figure 5 in de la Cruz Rodríguez et al. 2016).

\subsection{Energy Budget of IN Cancellations}

Our observations suggest that the process of IN flux cancellation provides a substantial amount of energy that is capable of heating the chromosphere locally. In what follows we estimate the energy content of IN canceling magnetic patches.

\subsubsection{Magnetic Energy}

Based on our tracking results, i.e., considering the number of detected canceling patches, their flux content, and the total $\mathrm{FOV}$, the flux cancellation rate is $27 \mathrm{Mx} \mathrm{cm}^{-2} \mathrm{day}^{-1}$, which is in agreement with the cancellation rate reported by Gošic et al. (2016).

To derive the magnetic field strength $(B)$ from the observed Stokes profiles in Mg $5173 \AA$, we carried out inversions using the SIR code (Ruiz Cobo \& del Toro Iniesta 1992). This code numerically solves the radiative transfer equation under the assumption of LTE and provides the temperature stratification, velocity, magnetic field strength, inclination, and azimuth angles along the line of sight. Inverting a nonLTE line with an LTE code means that the retrieved thermal parameters are not trustable, but the derived magnetic field stratification can be considered reliable (see Figure 14 in de la Cruz Rodríguez et al. 2012). We used three nodes in the temperature while the magnetic field strength and LOS velocity are assumed to have a linear gradient with height, which is necessary in order to fit the Stokes $V$ profiles that show an asymmetry between the blue and red lobes (for a detailed review, see del Toro Iniesta \& Ruiz Cobo 2016). The rest of the atmospheric parameters are kept constant with height. In order to reduce the number of free parameters, we assumed the magnetic filling factor to be equal to one and did not use stray-light contamination, which are reasonable assumptions when high-resolution observations are inverted as in this case. We use the Harvard Smithsonian Reference Atmosphere (Gingerich et al. 1971) as the initial model atmosphere. The average magnetic field strength of canceling IN patches (at the moment when cancellation starts) is found to be about $160 \mathrm{G}$, which is roughly in agreement with the average strength of IN fields reported earlier (e.g., $220 \mathrm{G}$; Orozco Suárez \& Bellot Rubio 2012a). This allows us to estimate the magnetic energy density per cancellation event:

$$
E_{\mathrm{mag}}=\frac{B^{2}}{8 \pi} \approx 1020 \mathrm{erg} \mathrm{cm}^{-3} .
$$

Considering that IN cancellations last about $t=3.6$ minutes at the photospheric level, we obtain an energy release rate of 

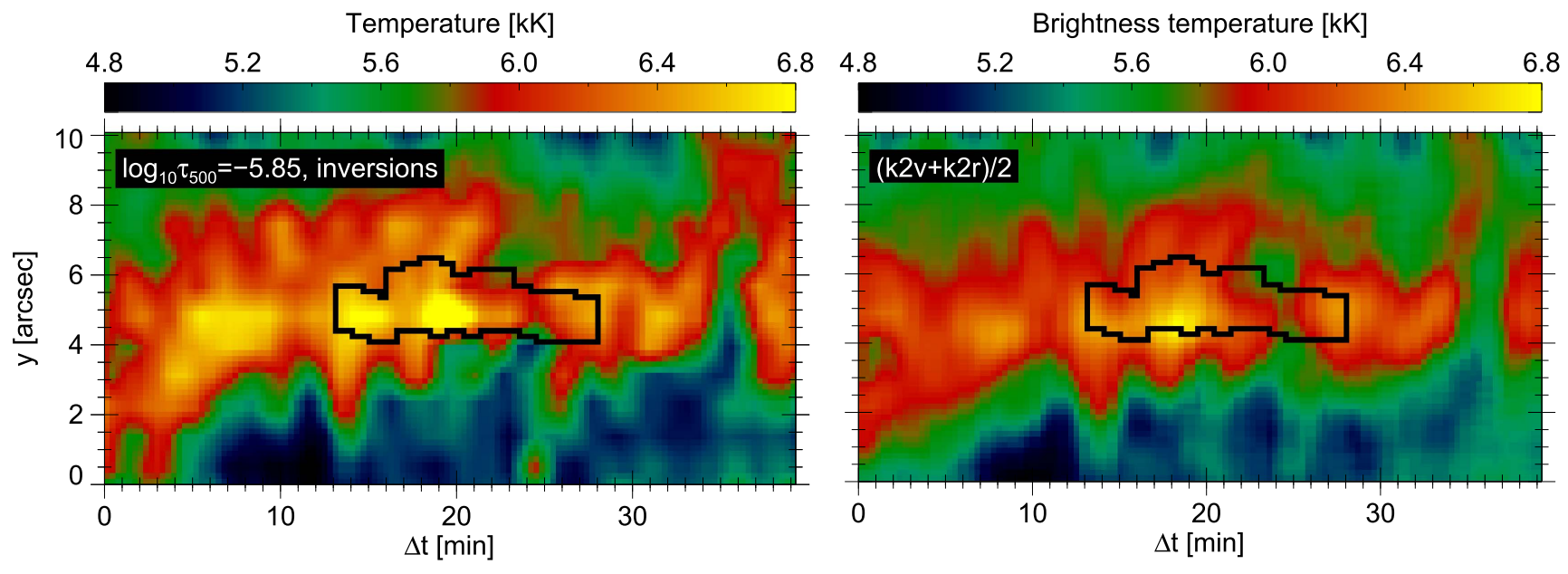

Figure 15. Left panel: temperature map at $\log _{10} \tau_{500}=-5.85$ derived from the inversions of the IRIS $\mathrm{Mg}$ II $h$ and $k$ lines in pixels at the cancellation site (black contour) and its surroundings. Right panel: the brightness temperature obtained from the average IRIS Mg II k2v and k2r peak intensities. $\Delta t=0$ minutes corresponds to $08: 41: 03$ UT.
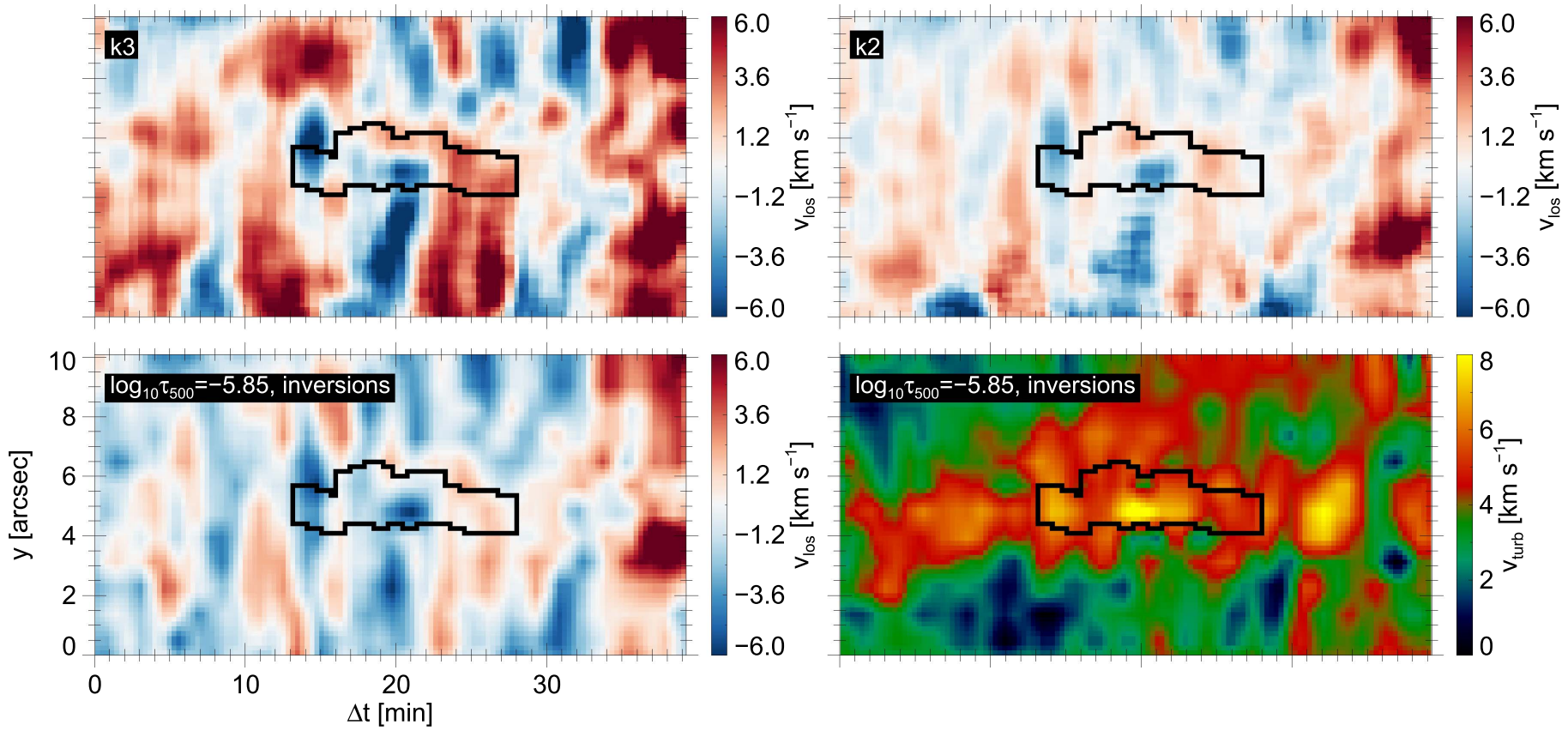

Figure 16. Reconstructed velocity maps associated with the temperature distribution from Figure 15. Upper left: LOS velocities derived from the Doppler shift of the IRIS Mg II $k_{3}$ line core. Upper right: LOS velocities obtained from the $\mathrm{Mg}$ II $k_{2}$ mean Doppler shifts. Lower left: reconstructed LOS velocities from inversions. Lower right: microturbulence velocities from inversions. The cancellation region is enclosed by the black contour.

$\Delta E_{\mathrm{mag}} \approx 4.7 \mathrm{erg} \mathrm{cm}^{-3} \mathrm{~s}^{-1}$. This shows that the energy accumulated inside individual cancellation regions is sufficient to locally balance the radiation loss of $10^{-1} \mathrm{erg} \mathrm{cm}^{-3} \mathrm{~s}^{-1}$ in the lower chromosphere (Vernazza et al. 1981).

However, taking into account the occurrence rate of cancellation events $(n=411)$ inside the observed FOV $\left(S_{\mathrm{FOV}}=1.2 \times 10^{19} \mathrm{~cm}^{2}\right)$, the horizontal extent of the reconnecting regions $\left(S_{\mathrm{r}}=0.6 \operatorname{arcsec}^{2}\right)$, and the duration of our observations $(T=2.6 \mathrm{hr})$, we can see that the energy contribution from IN cancellations averaged over time and area is $\Delta E_{\mathrm{mag}} n S_{\mathrm{r}} t / S_{\mathrm{FOV}} T \approx 1 \times 10^{-2} \mathrm{erg} \mathrm{cm}^{-3} \mathrm{~s}^{-1}$. In other words, the total energy input is an order of magnitude lower than what is necessary to globally maintain the chromospheric heating.

We want to stress here that our IRIS and SST observations clearly show that IN cancellations are local phenomena. This is also demonstrated with the time slice image shown in the right panel of Figure 17. As can be seen, the signal in IRIS SJI 1400 images is highly localized, and beyond the threshold of 60 counts (outlined by the white contour), drops rapidly to the background level. This indicates that the energy flux mostly propagates vertically and does not significantly affect the solar atmosphere in horizontal directions. Because of this and their small filling factor, the IN cancellation events that we study here (i.e., at the sensitivity levels of our current data set) seem to play a minor role in globally heating the IN chromosphere, despite the high local excess of energy flux.

\subsubsection{Missing Magnetic Energy}

We have established that the observed IN cancellations produce significant temperature increases in the chromosphere, but only locally. To play a global role in chromospheric 


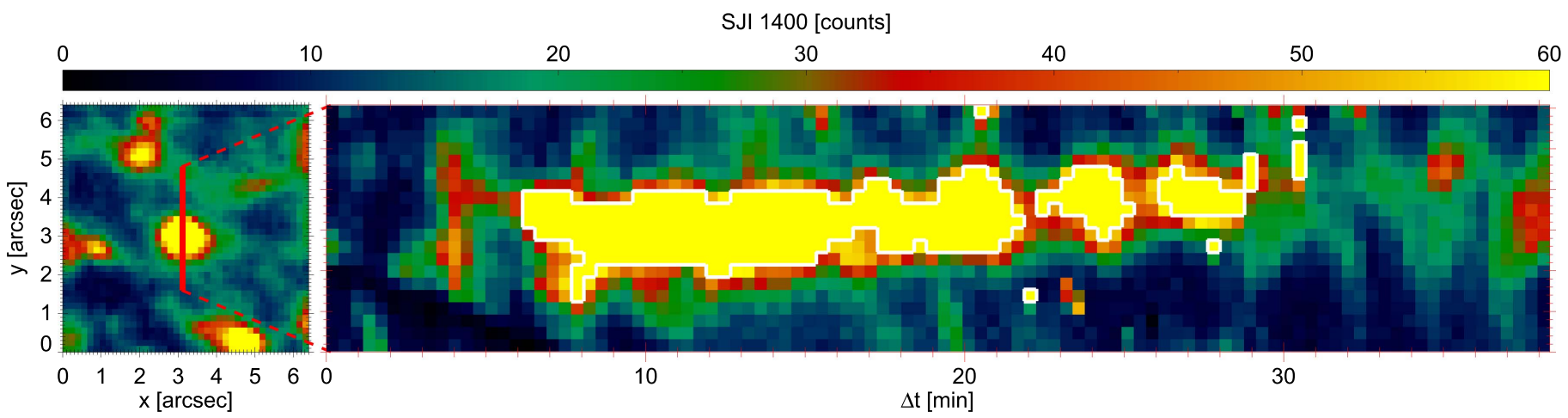

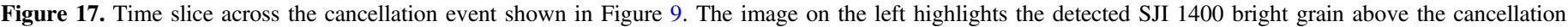

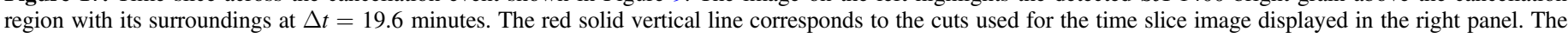
white contour coincides with the threshold of 60 counts used to detect bright structures in IRIS SJI 1400 filtergrams.

heating, a much larger area coverage would certainly be needed.

With the current data, we cannot exclude that cancellations occur ubiquitously, however. First, the sensitivity of our magnetograms is of the order of $24 \mathrm{G}$ ( 3 times the sigma level). In principle, it is possible that weaker fields are buried in the noise. Actually, very deep observations with the Hinode Spectro-Polarimeter (SP; Lites et al. 2013) have shown that the IN is nearly fully covered by weak fields (Bellot Rubio \& Orozco Suárez 2012; Orozco Suárez \& Bellot Rubio 2012a). If fields of $\sim 20 \mathrm{Mx} \mathrm{cm}^{-2}$ are present all over the solar surfaceas seems to be the case- and dissipate over the same timescales as the IN cancellations we observe with the SST, then they could explain the global heating of the IN chromosphere.

The challenge is thus to increase the sensitivity of the observations at least up to the level of the very deep Hinode/SP measurements and, at the same time, maintain high temporal resolution in order to detect the weakest IN fields, increase the surface area coverage, and investigate if they cancel out in a similar way to the stronger elements visible with the SST.

Only magnetic field measurements that are more sensitive can fully address the role of IN cancellations in the global heating of the IN chromosphere. The IRIS and SST observations analyzed here suggest that their area coverage is too small to provide the energy needed to sustain the chromosphere, but future measurements with the Daniel K. Inouye Solar Telescope (DKIST; Elmore et al. 2014) and the European Solar Telescope (EST; Collados et al. 2013) will allow more definitive statements to be made.

\section{Discussion and Conclusions}

In this paper, we studied cancellations of IN magnetic elements in an attempt to decipher their impact on the chromospheric and coronal heating. For this purpose, we carried out an analysis of simultaneous SST and IRIS observations of IN regions. Using SST magnetograms in the Mg 5173 A line, we identified all IN flux features that undergo either partial or total cancellations in the photosphere. Thanks to multiwavelength measurements, we were able to observe the temporal evolution of these magnetic elements and their coupling to the upper solar atmosphere. In particular, we analyzed SST and IRIS spectral line profiles at the locations where IN flux cancellations occur, sampling the solar atmosphere from the photosphere up to the transition regions.
Using SST Mg I $b_{2}$ magnetograms, we identified 411 cancellation events distributed all over the FOV. Most of them (360) involve IN flux concentrations and are covered by the IRIS FOV. In total, $76 \%$ of the detected events are cospatial with bright grains identified in IRIS SJI 1400 images, suggesting possible heating in the chromosphere. On average, cancellations seen in Mg magnetograms have shorter lifetimes than SJI 1400 bright structures, $\sim 3$ minutes compared to $\sim 12$ minutes, respectively. This result indicates that SJI 1400 bright grains above cancellation regions may be the result of reconnection of IN magnetic field lines in the chromosphere (and above) that starts earlier than the flux cancellations in the photosphere.

In fact, the interpretation of the photospheric flux cancellations as a consequence of magnetic reconnection in the chromosphere has been reported by multiple studies (e.g., Chae et al. 1998, 1999, 2010; Wang et al. 2000). The temporal evolution of the cancellation regions analyzed in this work implies that chromospheric reconnection is a plausible scenario for the quiet Sun IN cancellations. When two or more oppositepolarity patches that were not connected before are approaching toward each other, magnetic field lines start to reconnect at the chromospheric level. As a consequence, the released energy produces a local brightening in the chromosphere and possibly in the transition region. Such imprints are seen in both of our examples. In particular, we followed opposite-polarity flux patches as they approach. Before they start canceling in the photosphere, there are strong brightenings in the chromosphere as seen in the Ca II $8542 \AA$ intensity maps. They are located exactly above cancellation regions.

The spectral profiles of the Ca II $8542 \AA$ line have a very characteristic asymmetric form, clearly shifted toward the blue with the red wing in emission. Such a line shape has been reported to be associated with regions where strong local upflows are present and may be coupled with temperature enhancements caused by magnetic reconnections somewhere in the atmosphere (Ortiz et al. 2014; de la Cruz Rodríguez et al. 2015a). We obtained more evidence for the upflowing plasma inside cancellation regions from the properties of IRIS Mg II $h$ and $k$ lines, where according to Leenaarts et al. (2013b) a stronger $\mathrm{k} 2 \mathrm{r}$ peak implies upflow in the upper chromosphere. This is consistent with our $v_{\text {los }}$ derived from the non-LTE inversions of the $\mathrm{Mg}$ II $h$ and $k$ and UV triplet lines, shifts of the IRIS Mg II $k_{3}$ line cores, and from the Mg II $k_{2}$ mean Doppler shifts. We found that upflow velocities dominate inside cancellation regions. We also detected bright structures in IRIS 
SJI 1400 filtergrams, which supports the possibility of heating in the middle chromosphere and transition region since those filtergrams sample the region from the upper photosphere up to the transition region (Martínez-Sykora et al. 2015). During the latter phase of flux cancellation, dark fibrils appear in the core of the $\mathrm{H} \alpha$ line, which is likely a signature of plasma ejected upward by reconnection processes (Chae et al. 2010).

We have demonstrated that the magnetic energy density inside cancellation regions is sufficient to produce a local temperature increase in the chromosphere by more than $10^{3} \mathrm{~K}$. However, the area covered by cancellation events is not high enough to globally support the radiative losses necessary for chromospheric heating $\left(10^{-1} \mathrm{erg} \mathrm{cm}^{-3} \mathrm{~s}^{-1}\right.$; Vernazza et al. 1981). It is possible that numerous canceling magnetic elements are not visible in our observations due to limited spatial resolution and sensitivity. These fields would be too small and/or weak to produce polarization signals above the noise level. In such a case, we would lose their contribution and our calculated magnetic energies would be underestimated. To investigate the possible existence of IN fields on subresolution scales that contribute to chromospheric heating, we need measurements exceeding the capabilities of existing telescopes. The upcoming $4 \mathrm{~m}$ solar telescopes such as DKIST and EST will reach sensitivities of $10^{-4}$ and spatial resolutions of some $20 \mathrm{~km}$ with exposure times of a few seconds only. With such a sensitivity, we may be able to detect the weakest IN fields, while the unprecedented spatial resolution will help us detect fields below the currently accessible spatial and temporal scales.

In the meantime, according to our results, cancellations of IN flux patches play a role in the local energy balance in and around the cancellation sites, but cannot maintain global chromospheric heating by themselves, supporting the results of Wiegelmann et al. (2013) and Chitta et al. (2014). However, IN cancellations contribute alongside the other possible heating mechanisms. One such mechanism, for example, could be the heating of the upper solar atmosphere by the emergence of magnetic flux and the rise of the associated magnetic field lines up to the chromosphere and the transition region. This phenomenon will be addressed in our future work.

IRIS is a NASA small explorer mission developed and operated by LMSAL with mission operations executed at NASA Ames Research center and major contributions to downlink communications funded by ESA and the Norwegian Space Centre. M.G. was supported by NASA grant NNX16AC34G. J.dl.C.R. is supported by grants from the Swedish Research Council (2015-03994), the Swedish National Space Board (128/15), and the Swedish Civil Contingencies Agency (MSB). This project has received funding from the European Research Council (ERC) under the European Union's Horizon 2020 research and innovation programme (SUNMAG, grant agreement 759548). B.D.P. was supported by NASA grant NNX11AN98G and NASA contracts NNG09FA40C (IRIS). The work of L.B.R. and S.E.P. was supported by the Spanish Ministerio de Economía and Competitividad through grants ESP2013-47349-C6-1-R and ESP2016-77548-C5-1-R, including a percentage from European FEDER funds. Image reconstruction was performed at IAA-CSIC supercomputing facilities. The Swedish $1 \mathrm{~m}$ Solar Telescope is operated by the Institute for Solar Physics of Stockholm University in the Spanish Observatorio del Roque de los Muchachos of the Instituto de Astrofísica de Canarias.
M.C. was supported by the Research Council of Norway through its Centres of Excellence scheme, project number 262622, and through grants of computing time from the Programme for Supercomputing. This research has made use of NASA's Astrophysics Data System.

\section{ORCID iDs}

M. Gošić (ib https://orcid.org/0000-0002-5879-4371

J. de la Cruz Rodríguez (iD https://orcid.org/0000-00024640-5658

B. De Pontieu (iD https://orcid.org/0000-0002-8370-952X

L. R. Bellot Rubio (i) https://orcid.org/0000-0001-8669-8857

M. Carlsson (iD https://orcid.org/0000-0001-9218-3139

A. Ortiz (10) https://orcid.org/0000-0002-7212-2061

V. Polito (iD https://orcid.org/0000-0002-4980-7126

\section{References}

Barthol, P., Gandorfer, A., Solanki, S. K., et al. 2011, SoPh, 268, 1 Bellot Rubio, L. R., \& Orozco Suárez, D. 2012, ApJ, 757, 19

Carlsson, M., Leenaarts, J., \& De Pontieu, B. 2015, ApJL, 809, L30

Chae, J., Goode, P. R., Ahn, K., et al. 2010, ApJL, 713, L6

Chae, J., Qiu, J., Wang, H., \& Goode, P. R. 1999, ApJL, 513, L75

Chae, J., Wang, H., Lee, C.-Y., Goode, P. R., \& Schuhle, U. 1998, ApJL, 504, L123

Chitta, L. P., Kariyappa, R., van Ballegooijen, A. A., DeLuca, E. E., \& Solanki, S. K. 2014, ApJ, 793, 112

Collados, M., Bettonvil, F., Cavaller, L., et al. 2013, MmSAI, 84, 379

de la Cruz Rodríguez, J., Hansteen, V., Bellot Rubio, L. R., \& Ortiz, A. 2015a, ApJ, 810, 145

de la Cruz Rodríguez, J., Leenaarts, J., \& Asensio Ramos, A. 2016, ApJL, 830, L30

de la Cruz Rodríguez, J., Löfdahl, M. G., Sütterlin, P., Hillberg, T., \& Rouppe van der Voort, L. 2015b, A\&A, 573, A40

de la Cruz Rodríguez, J., Socas-Navarro, H., Carlsson, M., \& Leenaarts, J. 2012, A\&A, 543, A34

De Pontieu, B., Title, A. M., Lemen, J. R., et al. 2014, SoPh, 289, 2733

DeForest, C. E., Hagenaar, H. J., Lamb, D. A., et al. 2007, ApJ, 666, 576

del Toro Iniesta, J. C., \& Ruiz Cobo, B. 2016, LRSP, 13, 4

Elmore, D. F., Rimmele, T., Casini, R., et al. 2014, Proc. SPIE, 9147, 914707

Fontenla, J. M., Avrett, E. H., \& Loeser, R. 1993, ApJ, 406, 319

Galsgaard, K., \& Parnell, C. E. 2005, A\&A, 439, 335

Gingerich, O., Noyes, R. W., Kalkofen, W., \& Cuny, Y. 1971, SoPh, 18, 347

Gošić, M. 2015, PhD thesis, Instituto de Astrofísica de Andalucía (IAA-CSIC), Universidad de Granada, Spain, http://digibug.ugr.es/handle/10481/ 41677

Gošić, M., Bellot Rubio, L. R., del Toro Iniesta, J. C., Orozco Suárez, D., \& Katsukawa, Y. 2016, ApJ, 820, 35

Gošić, M., Bellot Rubio, L. R., Orozco Suárez, D., Katsukawa, Y., \& del Toro Iniesta, J. C. 2014, ApJ, 797, 49

Henriques, V. M. J. 2012, A\&A, 548, A114

Kosugi, T., Matsuzaki, K., Sakao, T., et al. 2007, SoPh, 243, 3

Kubo, M., Low, B. C., \& Lites, B. W. 2010, ApJ, 712, 1321

Leenaarts, J., Pereira, T. M. D., Carlsson, M., Uitenbroek, H., \& De Pontieu, B. 2013a, ApJ, 772, 89

Leenaarts, J., Pereira, T. M. D., Carlsson, M., Uitenbroek, H., \& De Pontieu, B. 2013b, ApJ, 772, 90

Lemen, J. R., Title, A. M., David, J. A., et al. 2012, SoPh, 275, 17

Lites, B. W., Akin, D. L., Card, G., et al. 2013, SoPh, 283, 579

Livi, S. H. B., Wang, J., \& Martin, S. F. 1985, AuJPh, 38, 855

Livingston, W. C., \& Harvey, J. 1975, BAAS, 7, 346

Löfdahl, M. G. 2002, Proc. SPIE, 4792, 146

Longcope, D. W., \& Kankelborg, C. C. 1999, ApJ, 524, 483

Martin, S. F. 1988, SoPh, 117, 243

Martínez González, M. J., \& Bellot Rubio, L. R. 2009, ApJ, 700, 1391

Martínez Pillet, V., Del Toro Iniesta, J. C., Álvarez-Herrero, A., et al. 2011, SoPh, 268, 57

Martínez-Sykora, J., Rouppe van der Voort, L., Carlsson, M., et al. 2015, ApJ, 803,44

Meyer, K. A., Sabol, J., Mackay, D. H., \& van Ballegooijen, A. A. 2013, ApJL, 770, L18

November, N., \& Simon, W. G. 1998, ApJ, 333, 427 
Orozco Suárez, D., \& Bellot Rubio, L. R. 2012, ApJ, 751, 2

Orozco Suárez, D., Katsukawa, Y., \& Bellot Rubio, L. R. 2012, ApJL, 758, L38

Ortiz, A., Bellot Rubio, L. R., Hansteen, V., de la Cruz Rodríguez, J., \& Rouppe van der Voort, L. 2014, ApJ, 781, 126

Pereira, T. M. D., Carlsson, M., De Pontieu, B., \& Hansteen, V. 2015, ApJ, 806, 14

Pereira, T. M. D., Leenaarts, J., De Pontieu, B., Carlsson, M., \& Uitenbroek, H. 2013, ApJ, 778, 143

Pesnell, W. D., Thompson, B. J., \& Chamberlin, P. C. 2012, SoPh, 275, 3

Priest, E. R. 1987, in Appearance and Disappearance of Magnetic Flux at the Solar surface, in The Role of Fine-Scale Magnetic Fields on the Structure of the Solar Atmosphere, ed. E.-H. Schröter, M. Vázquez, \& A. A. Wyller (Cambridge: Cambridge Univ. Press), 297

Priest, E. R., Heyvaerts, J. F., \& Title, A. M. 2002, ApJ, 576, 533

Rathore, B., \& Carlsson, M. 2015, ApJ, 811, 80

Requerey, I. S., del Toro Iniesta, J. C., Bellot Rubio, L. R., et al. 2017, ApJS, 229, 14

Requerey, I. S., Ruiz Cobo, B., Gošić, M., et al. 2018, A\&A, 610, A84

Ruiz Cobo, B., \& del Toro Iniesta, J. C. 1992, ApJ, 398, 375

Scharmer, G. B., Bjelksjo, K., Korhonen, T. K., Lindberg, B., \& Petterson, B. 2003, Proc. SPIE, 4853, 341

Scharmer, G. B., Narayan, G., Hillberg, T., et al. 2008, ApJL, 689, L69

Scherrer, P. H., Schou, J., Bush, R. I., et al. 2012, SoPh, 275, 207

Schrijver, C. J., \& van Ballegooijen, A. A. 2005, ApJ, 630, 552
Shine, R. A., Title, A. M., Tarbell, T. D., et al. 1994, ApJ, 430, 413

Solanki, S. K., Barthol, P., Danilović, S., et al. 2010, ApJL, 723, L127

Spruit, H. C., Title, A. M., \& van Ballegooijen, A. A. 1987, SoPh, 110, 115

Straus, T., Deubner, F.-L., \& Fleck, B. 1992, A\&A, 256, 652

Thornton, L. M., \& Parnell, C. E. 2010, SoPh, 269, 13

Title, A. M., Tarbell, T. D., Topka, K. P., et al. 1989, ApJ, 336, 475

Trujillo Bueno, J., Shchukina, N., \& Asensio Ramos, A. 2004, Natur, 430, 326

Tsuneta, S., Ichimoto, K., \& Katsukawa, Y. 2008, SoPh, 249, 167

Uitenbroek, H. 2001, ApJ, 557, 389

van Noort, M., Rouppe van der Voort, L., \& Löfdahl, M. G. 2005, SoPh, 228,191

van Noort, M. J., \& Rouppe van der Voort, L. H. M. 2008, A\&A, 489, 429

Vernazza, J. E., Avrett, E. H., \& Loeser, R. 1981, ApJS, 45, 635

Vissers, G., \& Rouppe van der Voort, L. 2012, ApJ, 750, 22

Wang, H., \& Zirin, H. 1987, SoPh, 115, 205

Wang, J., Li, W., Denker, C., et al. 2000, ApJ, 530, 1071

Welsch, B. T., \& Longcope, D. W. 2003, ApJ, 588, 620

Wiegelmann, T., Solanki, S. K., Borrero, J. M., et al. 2013, SoPh, 283, 253

Withbroe, G. L., \& Noyes, R. W. 1977, ARA\&A, 15, 363

Woodard, M. F., \& Chae, J. C. 1999, SoPh, 184, 239

Zhang, J., Lin, G., Wang, J., Wang, H., \& Zirin, H. 1998, SoPh, 178, 245

Zhao, M., Wang, J. X., Jing, C. L., \& Zhou, G. P. 2009, RAA, 9, 933

Zhou, G., Wang, J., \& Jin, C. 2010, SoPh, 267, 63

Zirin, H. 1985, AuJPh, 38, 961

Zwaan, C. 1987, ARA\&A, 25, 83 